Apidologie, 1983, 14 (3), 147-174.

\title{
LE SPECTRE POLLINIQUE DES MIELS DU QUÉBEC
}

\author{
Marie-José FELLER-DEMALSY \\ avec la collaboration technique de Johanne PARENT \\ Université du Québec à Rimouski, 300, avenue des Ursulines \\ Rimouski (Québec) G5L 3AI; Canada
}

\begin{abstract}
RESUMÉ
L'analyse pollinique a été effectuée sur 164 échantillons de miel des années 1977 et 1978 provenant des principales régions du Québec où se pratique l'apiculture. L'origine de l'échantillonnage est différente à $80 \%$ de celle de l'analyse effectuée sur les miels de 1974 et 1975 (Feller-Demalsy et Lamontagne, Apidologie, 1979, 10 (4), 313-340, La comparaison des deux séries d'analyses montre :

- une augmentation de la fréquence de plusieurs formes de pollen probablement attribuable à la fluctuation des cultures ou des conditions climatiques;

- que les espèces caractéristiques géographiques des régions sont confirmées et précisées; analyse;

- que Vicia cracca peut être ajoutée aux plantes d'importance majeure citées dans la précédente

- deux nouvelles caractéristiques géographiques, Rhus typhina pour l'Ouest de la province et Acer spicatum pour l'Est, en particulier le Bas-Saint-Laurent-Gaspésie.
\end{abstract}

\section{INTRODUCTION}

La présente étude fait suite à la publication «Analyse pollinique des miels du Québec (FEller-Demalsy et LAMONTAGNE 1979). Ce premier travail avait été effectué sur 206 échantillons de miels provenant de toutes les régions où se pratique l'apiculture au Québec. Quatre formes de pollen d'importance majeure y ont été relevées : Trifolium hybridum/repens, Solidago, des arbres fruitiers et Salix. Par ailleurs des indicateurs géographiques y ont été identifiés dont les principaux sont : Lythrum salicaria (surtout la rive Nord du Saint-Laurent), Sanguisorba canadensis et Cornus stolonifera (Est de la province), Lotus corniculatus (l'Ouest de la province), Fagopyrum sagittatum (région de Montréal et Mauricie), Melilotus (régions écartées 
du Saint-Laurent). L'esquisse de six grandes zones caractéristiques y a été effectuée : le Nord-Ouest (Nord-Ouest Québécois), le Centre-Nord (Saguenay - Lac-SaintJean), l'Ouest (Outaouais), le Centre-Ouest et le Sud-Ouest (sud-ouest de Montréal, Richelieu, nord de Montréal et Mauricie), le Centre-Est et Est (Québec et Bas Saint-Laurent-Gaspésie), le Sud-Est (Nicolet et cantons de l'Est).

Le présent travail est justifié, non seulement par l'échantillonage relativement restreint que comportait cette première recherche par rapport à l'étendue du territoire investigué, mais surtout, par l'absence de plusieurs comtés et la sous-représentation de certaines régions telles celle du Nicolet dont nous avons pu obtenir un échantillonnage plus valable.

L'objectif de ce travail est donc principalement par l'étude de miels de ruchers non encore touchés par notre recherche d'apporter des données complémentaires permettant de préciser, de confirmer ou éventuellement d'infirmer les conclusions de l'étude antérieure, mais aussi d'évaluer une évolution possible dans le temps de la flore des miels au Québec.

En outre des observations personnelles sur le terrain de la végétation du Québec nous a permis de palier partiellement à la carence de données phytogéographiques quantitatives nécessaires pour l'interprétation des résultats.

\section{MATÉRIELS ET MÉTHODES}

L'étude a porté sur 164 échantillons de miels récoltés pour un tiers en 1977 et les deux tiers en 1978, pour la plupart par les services d'inspection du ministère de l'Agriculture directement chez l'apiculteur. Ces échantillons proviennent à $80 \%$ de ruchers différents de ceux sur lesquels a porté la première étude. Sur les 74 comtés ou se pratique l'apiculture, 49 sont représentés dans ce travail et 10 d'entre eux ne figurent pas dans la première analyse. Les deux études au total donnent un aperçu de 67 comtés. L'échantillonnage est meilleur pour 10 comtés ce qui donne une meilleure représentation de 5 régions qui sont celles de Québec (rive Sud), Nicolet, des Cantons de l'Ext, du Richelieu et du Sud-Ouest de Montréal. Cependant pour les régions du Nord de Montréal et de Québec (rive Nord) le nombre d'échantillons de cette seconde étude étant réduits, les résultats isolés devront être considérés avec prudence, ou seulement en comparaison avec ceux de la première étude. Par ailleurs ne disposant d'aucun miel du Nord-Ouest québécois ni de Beauce pour les années 1977 et 1978, ces régions ne seront pas directement étudiées dans le présent travail. Le tableau 1 et la figure 1 donnent la répartition de l'échantillonnage. Les limites de comtés ont été déterminées d'après le Répertoire toponymique du Québec (Commission de Toponymie 1978). Pour la facilité de l'étude, les comtés ont été regroupés en régions qui correspondent aux régions agricoles (d'après les données du Bureau de la Statistique du Québec 1977 et 1978). Comme dans l'étude précédente, la région de Québec a cependant été divisée en deux parties, la rive Nord et la rive Sud du Saint-Laurent qui paraissent différentes au point de vue de l'environnement et des caractéristiques géologiques et géographiques.

Les échantillons ont été traités selon la méthode de la Commission Internationale de Botanique apicole décrite par LouveauX, Maurizıo et Vorwohl (1970) de la même façon que dans notre étude de 1979. Les spectres ont été établis à partir de 200 grains de pollen. Les indicateurs de miellat n'ont pas été pris en considération. 
SPECTRE POLLINIQUE DES MIELS DU QUÉBEC

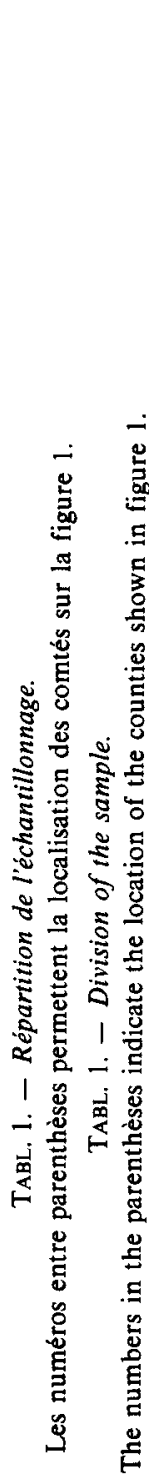

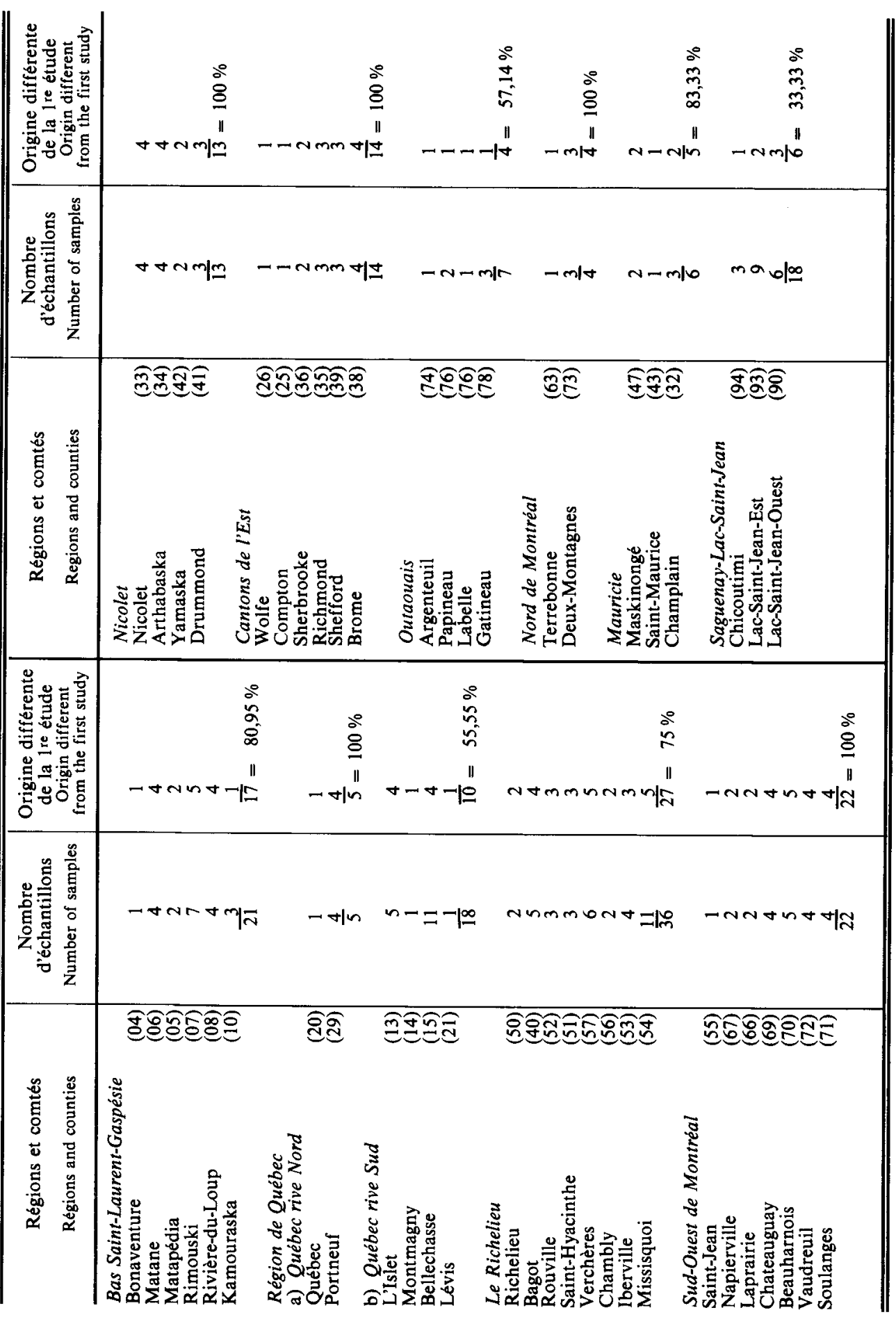


Fio. 1. - Distribution géographique des échantillons étudiés.

Chaque numéro correspond à un comté (voir tableau 1).

- Site d'échantillonnage $74-75$

- Site d'échantillonnage 77-78

Zone agricole

Limite de comtés

Limite de régions

-... Limite interprovinciale

_.__ Limite internationale

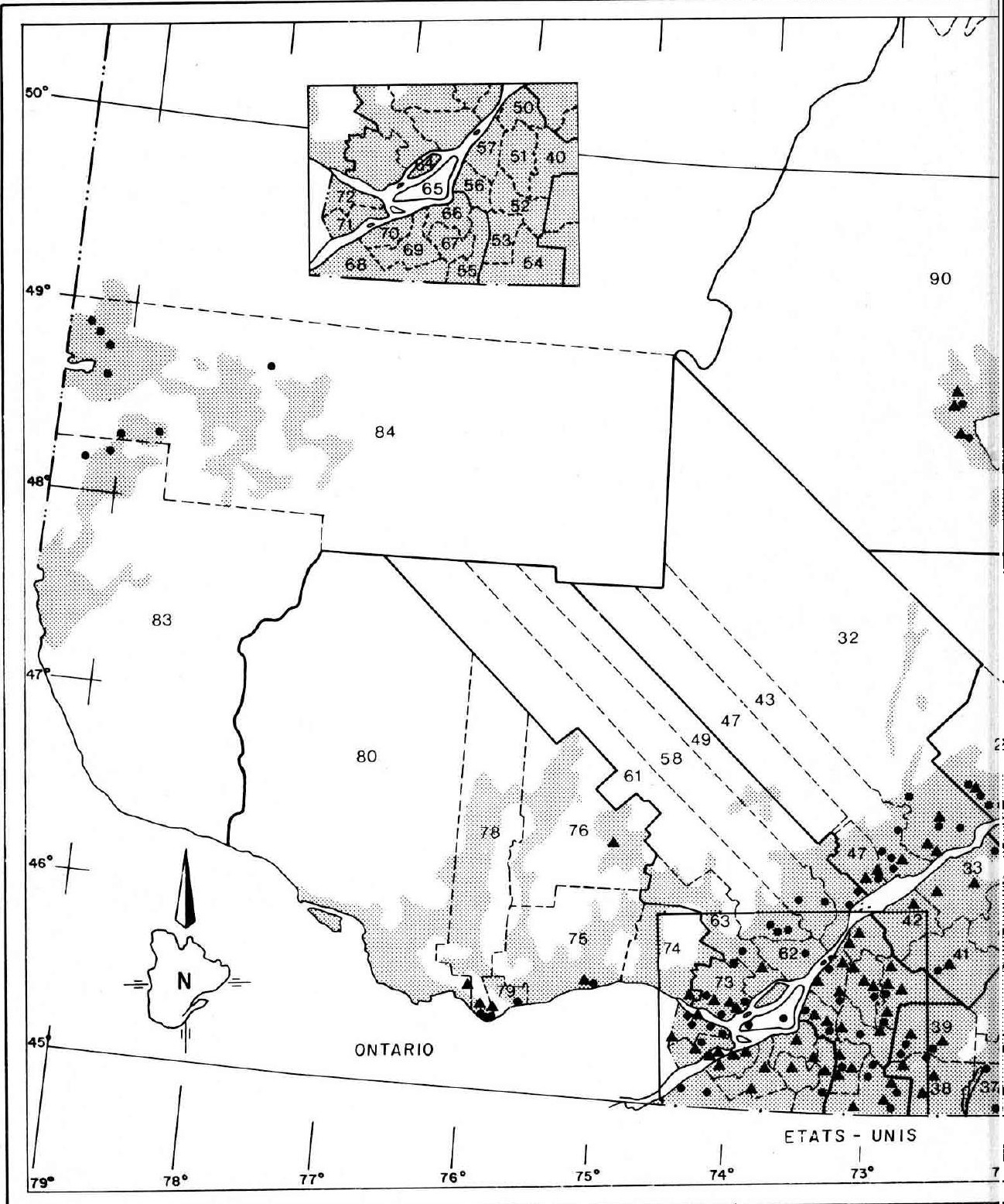


FIG. 1. - Geographic distribution of the samples studied.

Each number corresponds to a county (see Table 1)

- Site of samples 7475

- Site of samples 77-78

Agricultural zone

Border of the counties

Border of the regions

Border of the provinces

- - International boundaries

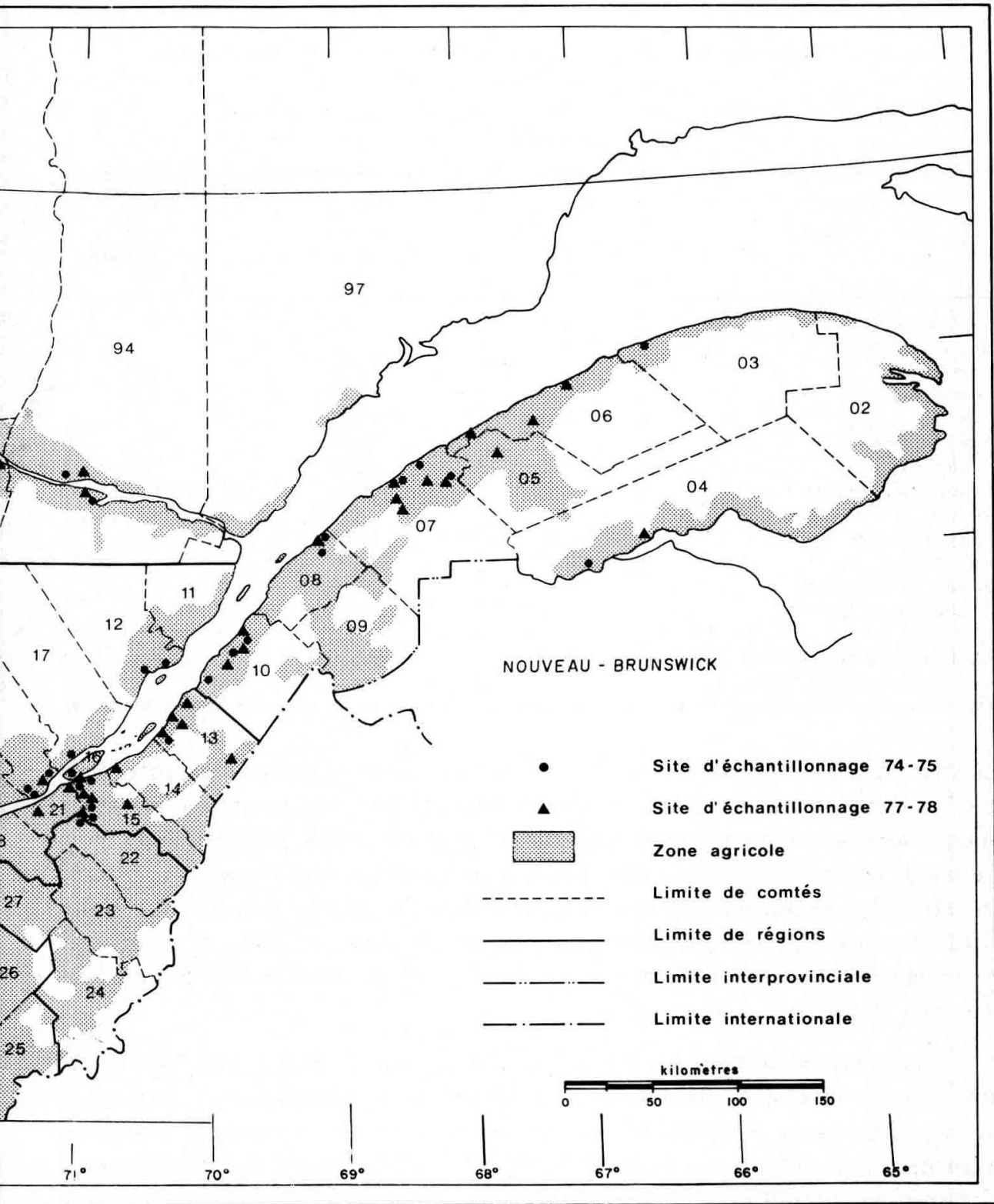




\section{RÉSULTATS}

\section{Le nombre de grains de pollen dans les miels}

Les nombres de grains de pollen par $10 \mathrm{~g}$ de miel se situent principalement dans les nombres moyens bien que presque autant d'échantillons de miels soient pauvres en grain (tableau 2) : $40 \%$ possèdent moins de 20000 grains, $56 \%$ seulement

TABL. 2. - Nombre de grains de pollen dans les miels du Québec et de chaque région : pourcentage de chaque catégorie.

TABL. 2. - Number of pollen grains in the honey of Québec and each region : percentage of each category.

\begin{tabular}{|c|c|c|c|}
\hline $\begin{array}{l}\text { Régions } \\
\text { Regions }\end{array}$ & $\begin{array}{c}\text { Pauvre } \\
\text { Low } \\
(<20000) \\
(\%)\end{array}$ & $\begin{array}{c}\text { Moyen } \\
\text { Medium } \\
(20000-100000) \\
(\%)\end{array}$ & $\begin{aligned} & \text { Riche } \\
& \text { High } \\
(> & 100000) \\
& (\%)\end{aligned}$ \\
\hline $\begin{array}{l}\text { Bas St-Laurent-Gaspésie } \\
\text { Région de Québec } \\
\text { - rive Nord } \\
\text { - rive Sud } \\
\text { Nicolet } \\
\text { Cantons de l'Est } \\
\text { Le Richelieu } \\
\text { Sud-Ouest de Montréal } \\
\text { Outaouais } \\
\text { Nord de Montréal } \\
\text { La Mauricie } \\
\text { Saguenay-Lac-Saint-Jean } \\
\text { Moyenne pour le Québec }\end{array}$ & $\begin{array}{l}57,14 \\
\\
20,00 \\
38,89 \\
20,77 \\
35,71 \\
50,00 \\
36,36 \\
14,29 \\
50,00 \\
50,00 \\
28,22 \\
39,63\end{array}$ & $\begin{array}{l}42,86 \\
\\
60,00 \\
55,56 \\
69,13 \\
64,29 \\
44,44 \\
59,09 \\
71,43 \\
25,00 \\
50,00 \\
77,28 \\
56,10\end{array}$ & $\begin{array}{c}- \\
20,00 \\
5,56 \\
- \\
- \\
5,56 \\
4,55 \\
14,29 \\
25,00 \\
- \\
\overline{4} \\
4,27\end{array}$ \\
\hline $\begin{array}{l}\text { Moyenne des } 2 \text { études pour le Québec } \\
\text { Average of the two studies for Quebec }\end{array}$ & 48,11 & 47,57 & 4,32 \\
\hline
\end{tabular}

dépassent les 100000 grains. Ces chiffres sont très proches de ceux du précédent travail et une moyenne entre les 2 études montre que les miels pauvres en grains de pollen et les miels à nombre moyen de grains se retrouvent en définitive à peu près en quantité égale, les miels à nombre élevé de grains constituant l'exception. Malgré la diversité de traitements des miels au Québec déjà signalée précédemment et toute la prudence nécessitée par l'interprétation de ces résultats, ces données pourraient être retenues comme caractéristiques au Québec, quelques fluctuations pouvant cependant se présenter autour d'elles.

Comme précédemment, les miels à nombre moyen de grains sont plus abondants dans les régions du Sud-Ouest de Montréal, de l'Outaouais et du SaguenayLac-Saint-Jean, mais le présent échantillonnage en montre en outre dans les régions de Québec, de Nicolet et des Cantons de l'Est. Les miels riches en grains de pollen se rencontrent surtout dans le Sud-Ouest et dans l'Ouest de la province. 


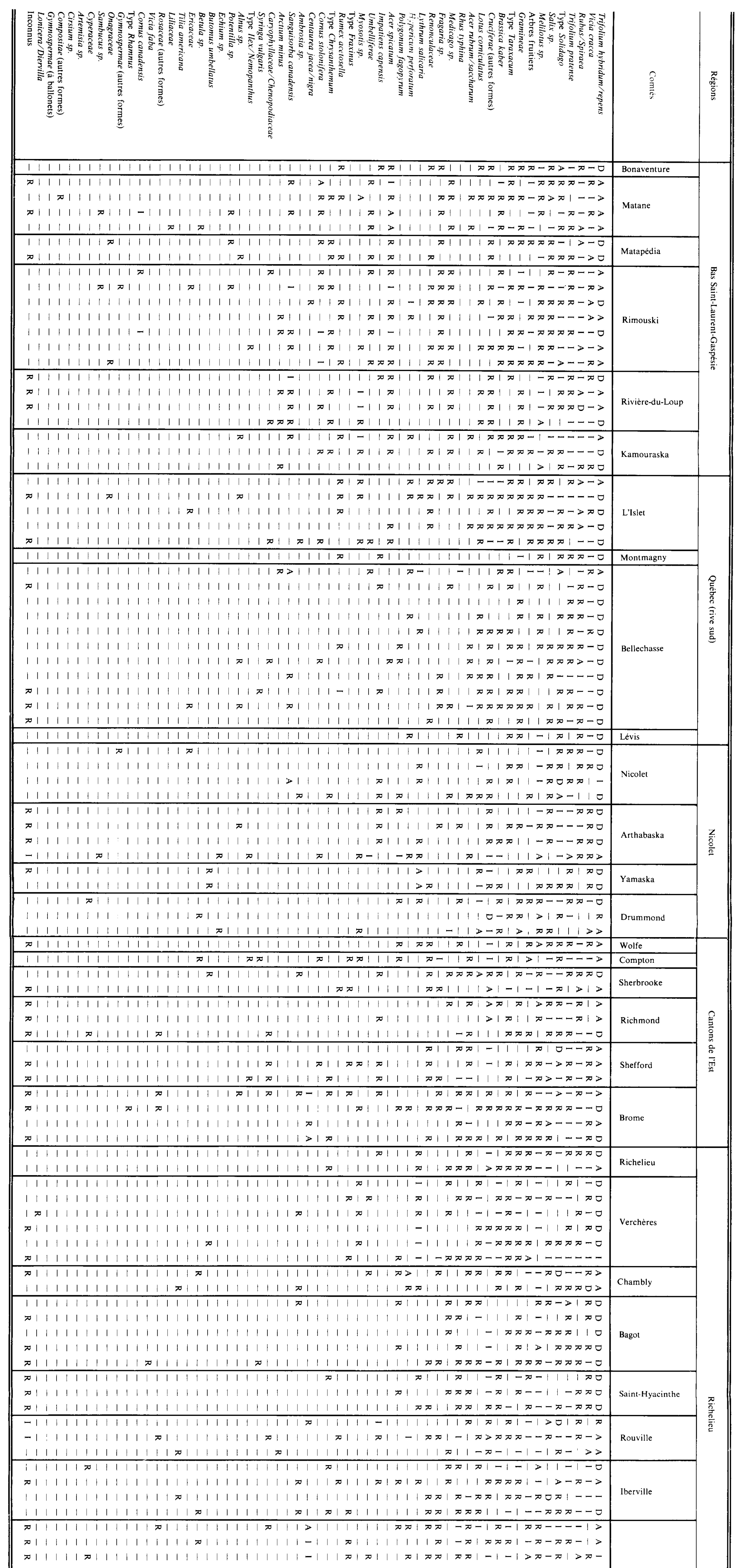




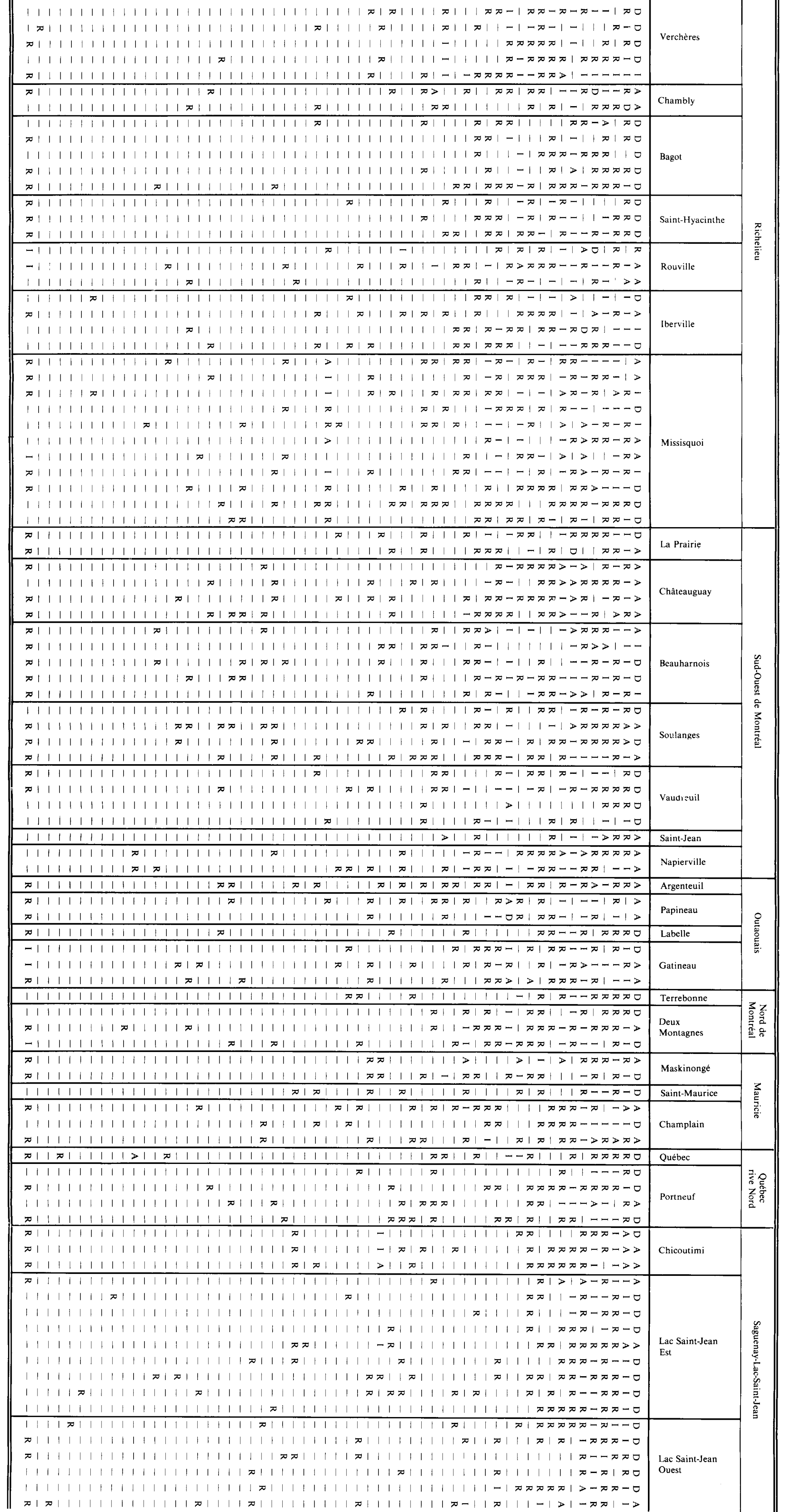

TABL. 3. - Spectre pollinique des 164 échantillons de miels.

D : pollen dominant $(>45 \%)$

A : pollen d'accompagnement $(16<45 \%)$

I : pollen isolé important (3-15\%)

: pollen isole important $(3-15 \%)$

TABL. 3. - Pollen spectrum of 164 honey samples

$D$ : dominant pollen $(>45 \%)$

A : secondary pollen $(16-45 \%)$

$R:$ minor pollen $(<3 \%)$ 


\section{Le nombre de formes de pollen}

Cinquante-sept formes de pollen ont été identifiées. Certaines formes isolées, parmi les plus rares décelées précédemment n'ont pas été retrouvées et paraissent accidentelles ou leur fréquence extrêmement réduite. Par ailleurs, ont été identifiées plusieurs formes nouvelles dont quelques unes constituent un apport intéressant pour les miels de certaines régions.

Le tableau 3 donne le spectre pollinique des échantillons analysés, ordonnés par comtés et regroupés par régions. Les formes de pollen qui n'appartiennent pas avec certitude à une espèce ou à un genre déterminé, ont été regroupés sous le nom de la famille (Cruciferae autres formes, Graminae, Umbelliferae, Caryophyllaceae/Chenopodiaceae, Ericaceae, Rosaceae autres formes, Gymnospermes à ballonnets et autres formes, Onagraceae) ou d'une forme type apparemment plus fréquente (type Solidago, type Taraxacum, type Chysanthemum, Type Fraxinus, type Ilex/Nemopanthus, type Rhamnus). C'est le cas aussi pour les formes de certains genres ou de certaines espèces qui ont été regroupées (Trifolium hybridum/repens, Centaurea jacea/nigra, Acer rubrum/saccharum, Lonicera/Diervilla). Il est probable que le relevé de la forme Rubus renferme quelques grains du genre Spiraea, toujours en quantité minime mais parfois difficilement discernables par suite de déformations subies dans les miels. La dénomination arbres fruitiers inclut outre les genres Pyrus $s p$. et Prunus sp. aussi les genres Crataegus sp. et Amelanchier sp. Plusieurs formes inconnues ont été relevées et répertoriées, mais chacune ne se trouve qu'à l'état de pollen isolé et n'atteint au maximum $3 \%$ que dans 3 miels. Le nombre de formes inconnues est inférieur à une par miel en moyenne $(0.78)$.

Les nombres de formes de pollen par miel (voir tableau 4) se situent dans les catégories se classant entre 6 et 25 formes avec le plus grand nombre de maxima entre 16 et 20 formes qui correspond d'ailleurs au maximum des moyennes québécoises. La moyenne du nombre de formes s'élève pour le Québec à 15,34 formes par miel (les différentes inconnues incluses). Des moyennes inférieures se retrouvent dans la région de Québec, de Nicolet (la plus basse), du Richelieu et du SaguenayLac-Saint-Jean, des moyennes supérieures dans les autres régions, la plus élevée étant dans l'Outaouais. Le nombre de formes de pollens par miel est plus élevé dans cet échantillonnage que dans le précédent. Cette différence ne résulte pas seulement de l'étude d'un plus grand nombre de grains par miel (200 au lieu de 100) ni de l'adjonction dans les comptages des différentes formes d'inconnues. L'étude du deuxième échantillonnage effectué dans les mêmes conditions que le premier montre un maximum moyen de 11-15 formes pour la province alors qu'il était dans les 6-10 formes dans le précédent échantillonnage. Le nombre moyen de formes de pollen par miel dans ces mêmes conditions s'élèverait à 11,76 au lieu de 9,43. Cette augmentation doit donc provenir de la différence d'échantillonnage, qu'elle soit dans l'espace ou dans le temps. 
FIG. 2. - Spectre de fréquence dans les miels étudiés (en \% des échantillons analysés).

Les formes de pollen se rapportant à une famille ont été placées dans la catégorie nectarifère ou pollinifère pour peu qu'une espèce soit présumée nectarifère ou pollinifère;

dans le cas de type, seule cette espèce a été considérée pour ces propriétés.
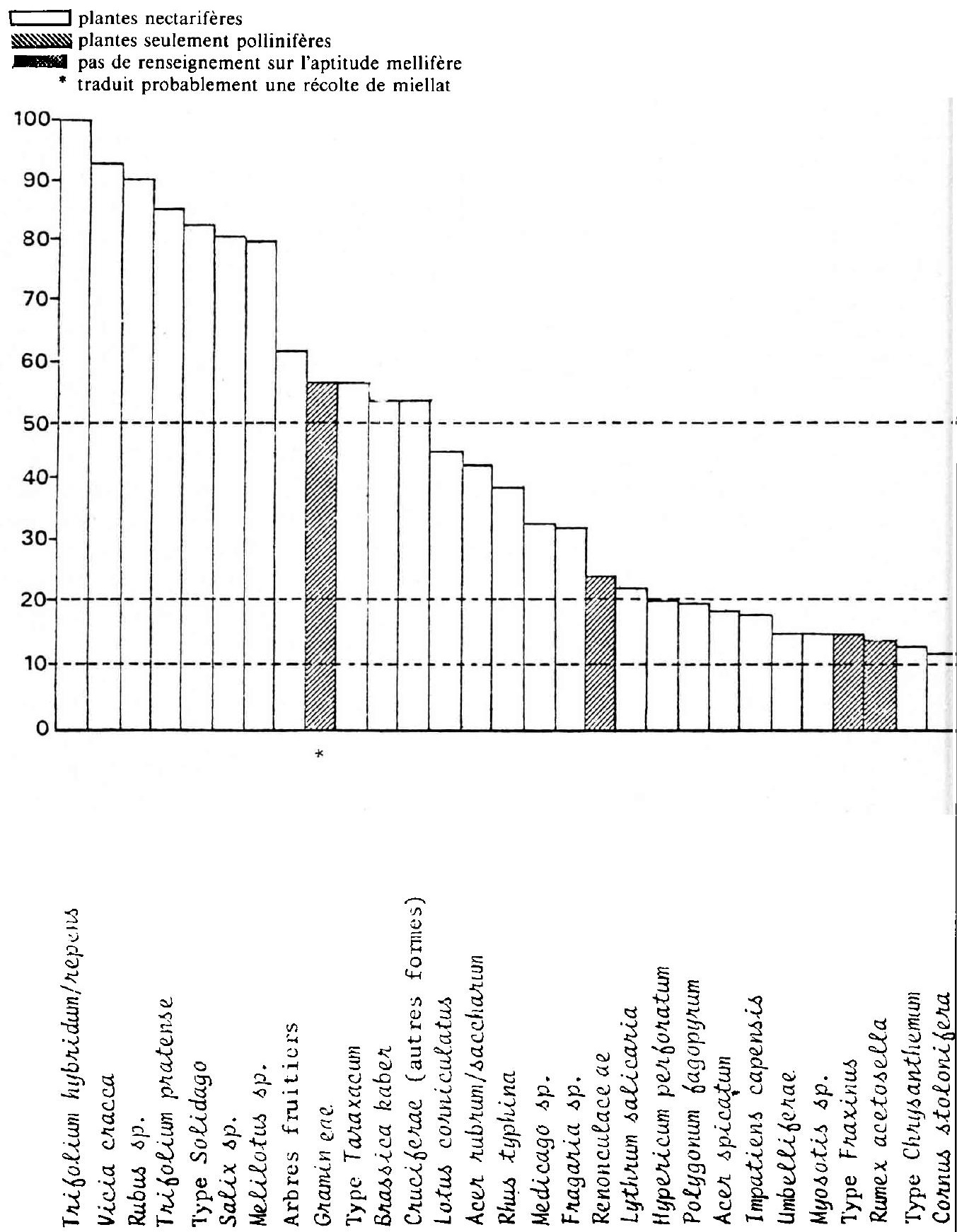
FIG. 2. - Frequency spectrum of the honeys studied (in \% of samples analyzed). The pollen forms in relation to the family were placed in the category nectariferous or polleniferous if only one specie was presumed to be nectariferous or pollineferous; in the case of type, only this specie was considered for these qualities.

nectariferous plants

plants only for pollen

no data on the disposition by bees

probably represents a collection of honeydew.
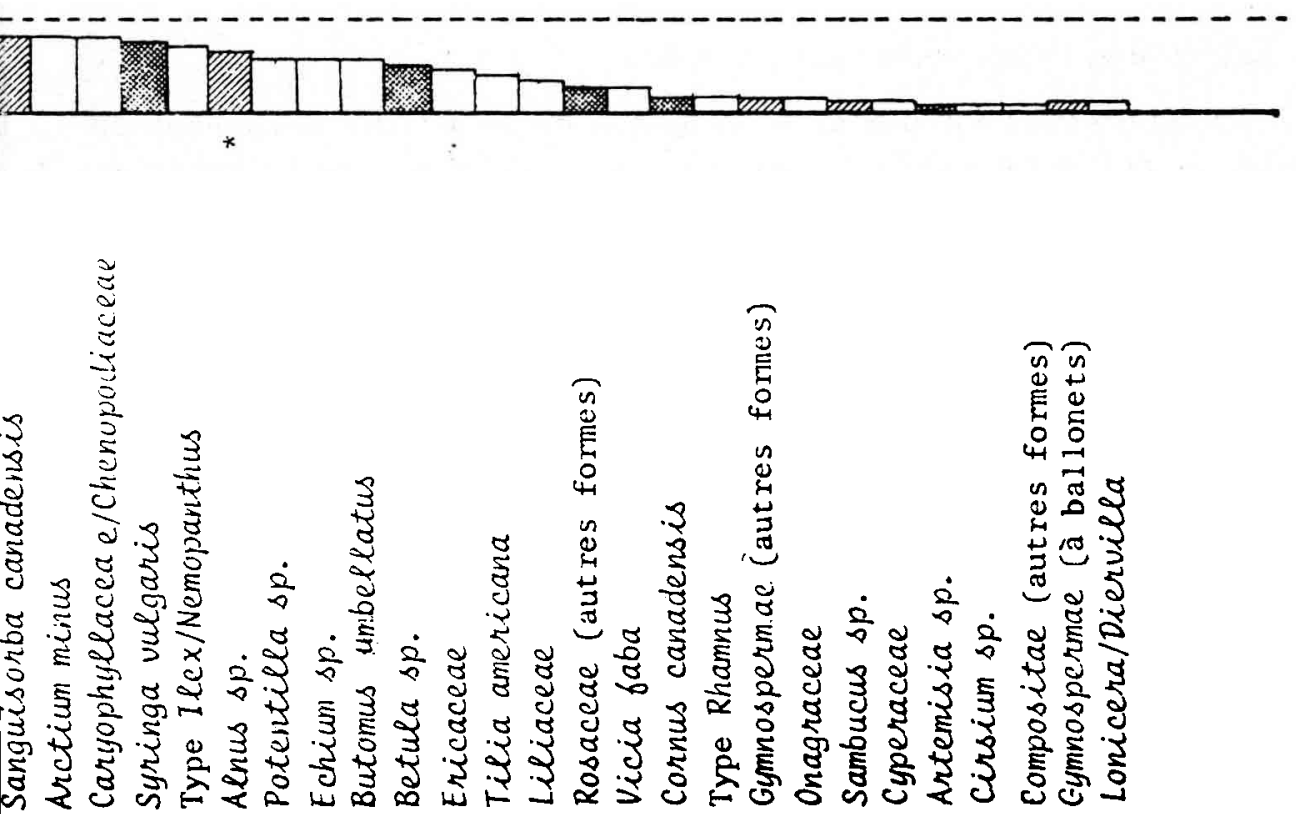
TABL. 4. - Pourcentage des différentes catégories de nombre de formes de pollen par miel et nombres mojens de formes de pollen par miel évalués par région et pour le Québec

TABL. 4. - Percentage of different categories of number of pollen forms in honey evaluated for the regions and Quebec.

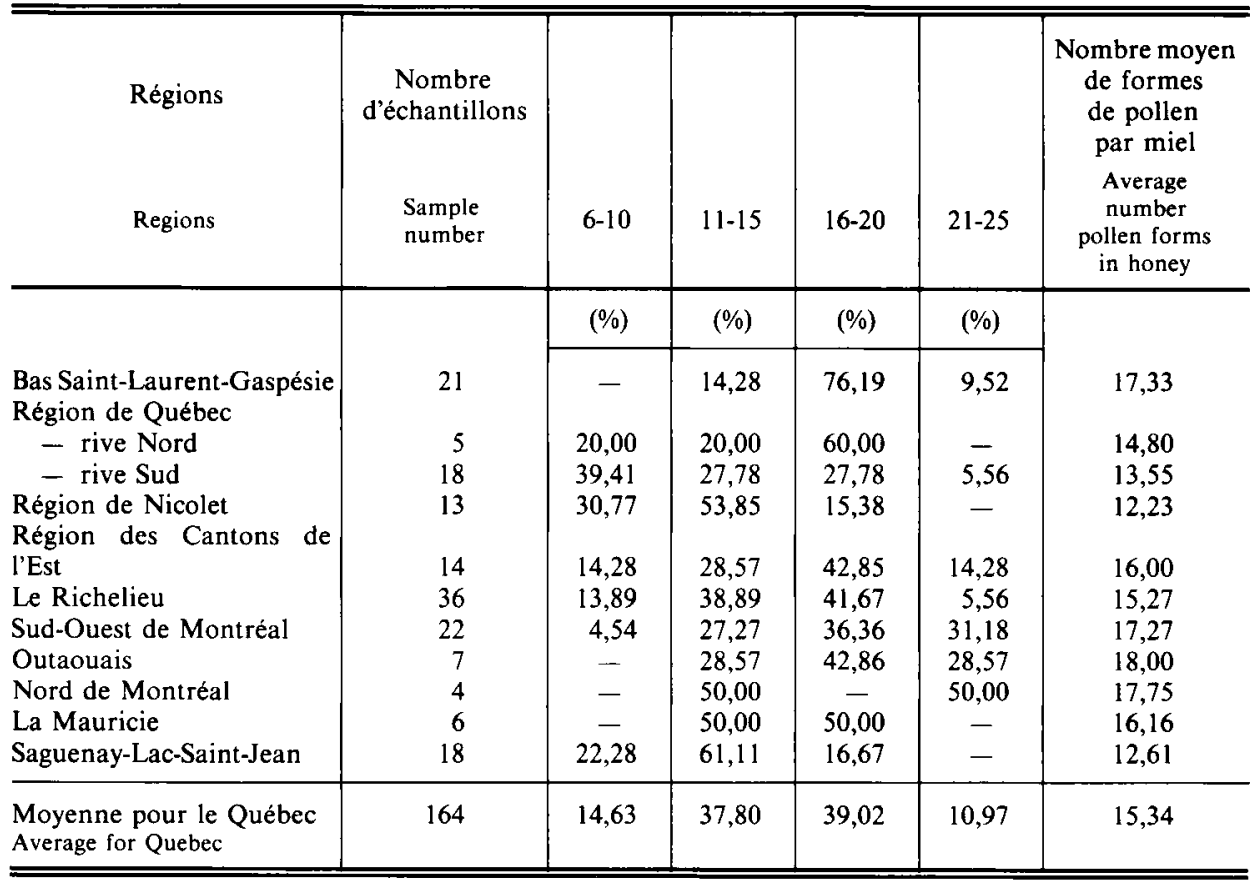

\section{Le spectre de fréquence des formes de pollen}

Les formes très fréquentes présentes dans au moins $50 \%$ des échantillons sont (figure 2) : Trifolium hybridum/repens (trèfle alsike/trèfle blanc) (100\%), Vicia cracca (vesce jargeau) $(92,68 \%$ ), Rubus sp. (framboisier) $(87,88 \%$ ), Trifolium pratense (trèfle rouge) $(84,76 \%)$, type Solidago (verge d'or) $(82,32 \%)$, Salix sp. (Saule) $(80,89 \%)$, Melilotus sp. (melilot) (79,88\%), arbres fruitiers (62,19\%), Graminées (56,75\%), type Taraxacum (pissenlit) (56,75\%), Brassica kaber (moutarde des champs) $(53,65 \%)$ et autres Crucifères $(53,65 \%)$. Toutes ces plantes sont nectarifères, pollinifères et entomophiles sauf les Graminées qui sont seulement pollinifères. Les formes fréquentes, présentés dans $20-50 \%$ des échantillons sont : Lotus corniculatus (lothier corniculé) (45,12\%), Acer rubrum/saccharum (Erable rouge/à sucre) (43,29\%), Rhus typhina (vinaigrier) $(39,02 \%)$, Medicago sp. (luzerne) (33,54\%), Fragaria sp. (fraisier) (32,93\%), Renonculacées (25,00\%), Lythrum salicaria (salicaire) $(23,17 \%)$, Hypericum perforatum (Millepertuis perforé) (20,73\%), Polygonum fagopyrum (sarrasin) $(20,12 \%)$. La plupart sont nectárifères, pollinifères et entomophiles. Parmi les Renonculacées, Anemone sp. est pollinifère. Les formes peu fréquentes (10-20\%) sont : Acer spicatum (érable en épis), Impatiens capensis (impatiente du cap ou chou 
sauvage), des Ombellifères, Myosotis sp., le type Fraxinus (frêne), le type Chrysanthemum (marguerite), Rumex acetosella (petite oseille), Cornus stolonifera (Hart rouge), Centaurea jacea/nigra (Centaurée jacée/noire) et Ambrosia sp. (Herbe à poux). La plupart sont nectarifères, pollinifères et entomophiles. Rumex acetosella est pollinifère et anémophile et Fraxinus et Ambrosia pollinifères. Parmi les pollens rares, se situent 26 formes dont plusieurs sont nectarifères et (ou) pollinifères.

Le nombre de formes très fréquentes est plus important dans ce $2^{e}$ échantillonnage que dans le premier. Douze formes atteignent la fréquence de $50 \%$ au lieu de

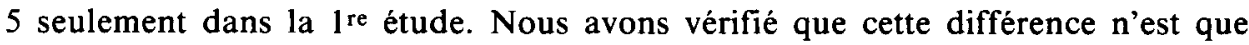
partiellement attribuable à l'étude d'un plus grand nombre de grains par miel (200 au lieu de 100). Une étude portant sur 100 grains par miel a montré que 8 formes atteignent encore cette fréquence, les Graminées, le type Taraxacum, Brassica kaber et les autres Crucifères restant alors au niveau de pollen fréquent.

Le tableau 5 qui donne l'indice de fréquence (rapport de la fréquence régionale et de la fréquence moyenne du Québec) exprime les différences régionales de fréquence des pollens par rapport à la moyenne québécoise. Les trois premières formes de la première catégorie, les pollens très fréquents et le type Solidago sont répartis à peu près uniformément dans toutes les régions du Québec. Quelques variations se présentent pour les autres formes de la même classe, mais c'est surtout au niveau des autres catégories de fréquence que se dessinent des disparités de répartition de formes entre les régions.

\section{L'abondance des formes de pollen dans les miels.}

Le tableau 6 donne des formes de pollen les plus abondantes classées par ordre de fréquence. Toutes les catégories de fréquence sont représentées : presque toutes les formes très fréquentes, environ $50 \%$ des formes fréquentes et peu fréquentes et deux formes rares. Des pollens dominants ( $>45 \%$ ) se rencontrent chez plusieurs formes très fréquentes: Trifolium hybridum/repens, Vicia cracca, Rubus, le type Solidago, Salix, Melilotus et les Crucifères; un cas chez les formes fréquentes : Lotus corniculatus. A part Trifolium hybridum/repens et le type Solidago, les cas de dominance sont isolés.

\section{DUSCUSSION ET CONCLUSION}

Des 12 formes très fréquentes mises en évidence par cet échantillonnage, cinq seulement atteignent cette catégorie de fréquence au niveau régional. Il s'agit de Trifolium hybridum/repens, Vicia cracca, Rubus, type Solidago et Melilotus. Trois de ces formes remplissaient les mêmes conditions dans le ler échantillonnage : Trifolium hybridum/repens, Vicia cracca et le type Solidago qui paraissent des caractéristiques stables et générales pour les miels de toutes les régions du Québec. La fréquence de ces formes est restée stable également au niveau de la province, ce qui n'est pas le 

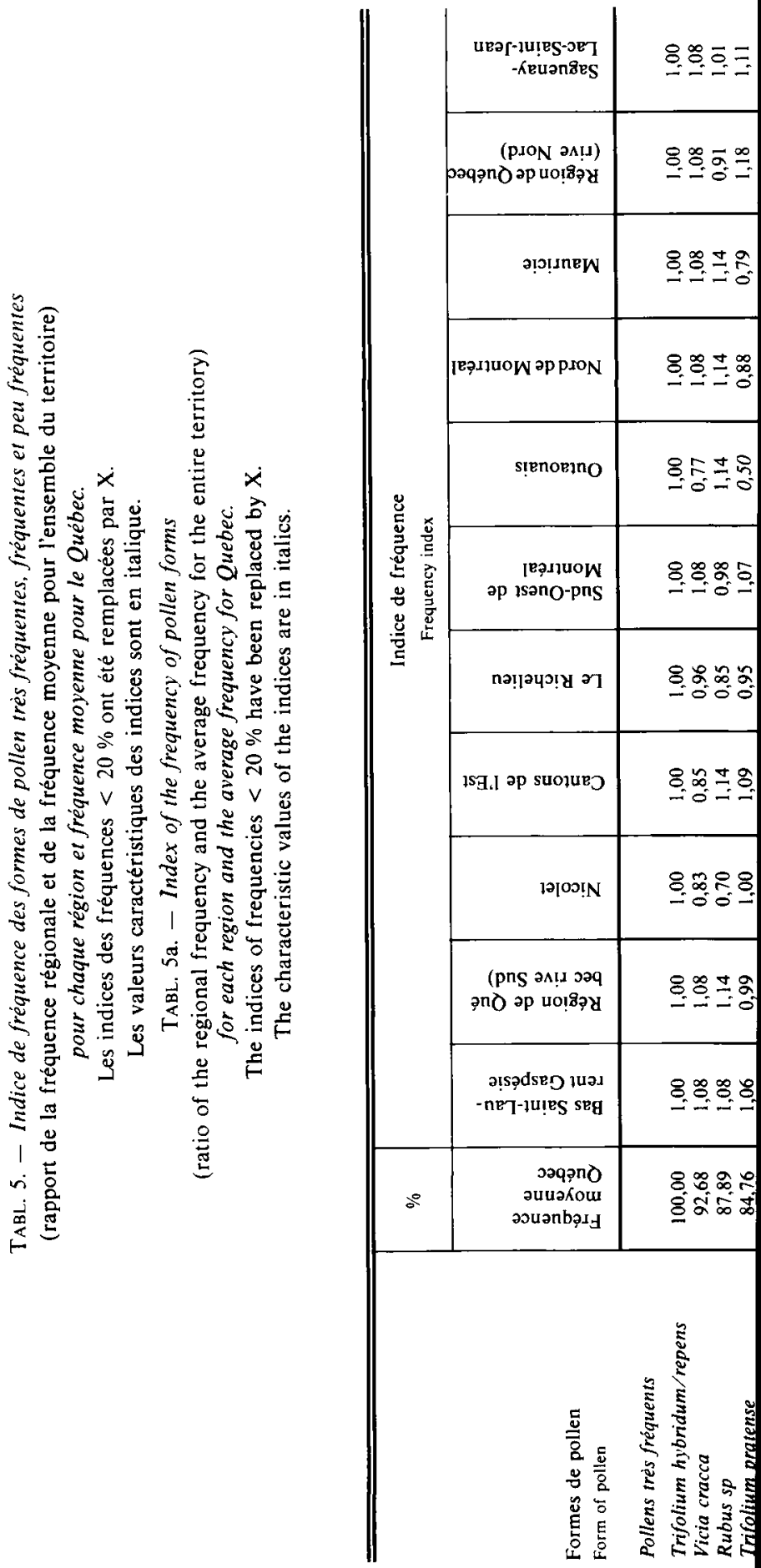
TABL. 6. - Nombre de pollens dominants (premier chiffre) et d'accompagnement (second chiffre) dans les miels du Québec et de chaque région classés par ordre de fréquence décroissante.

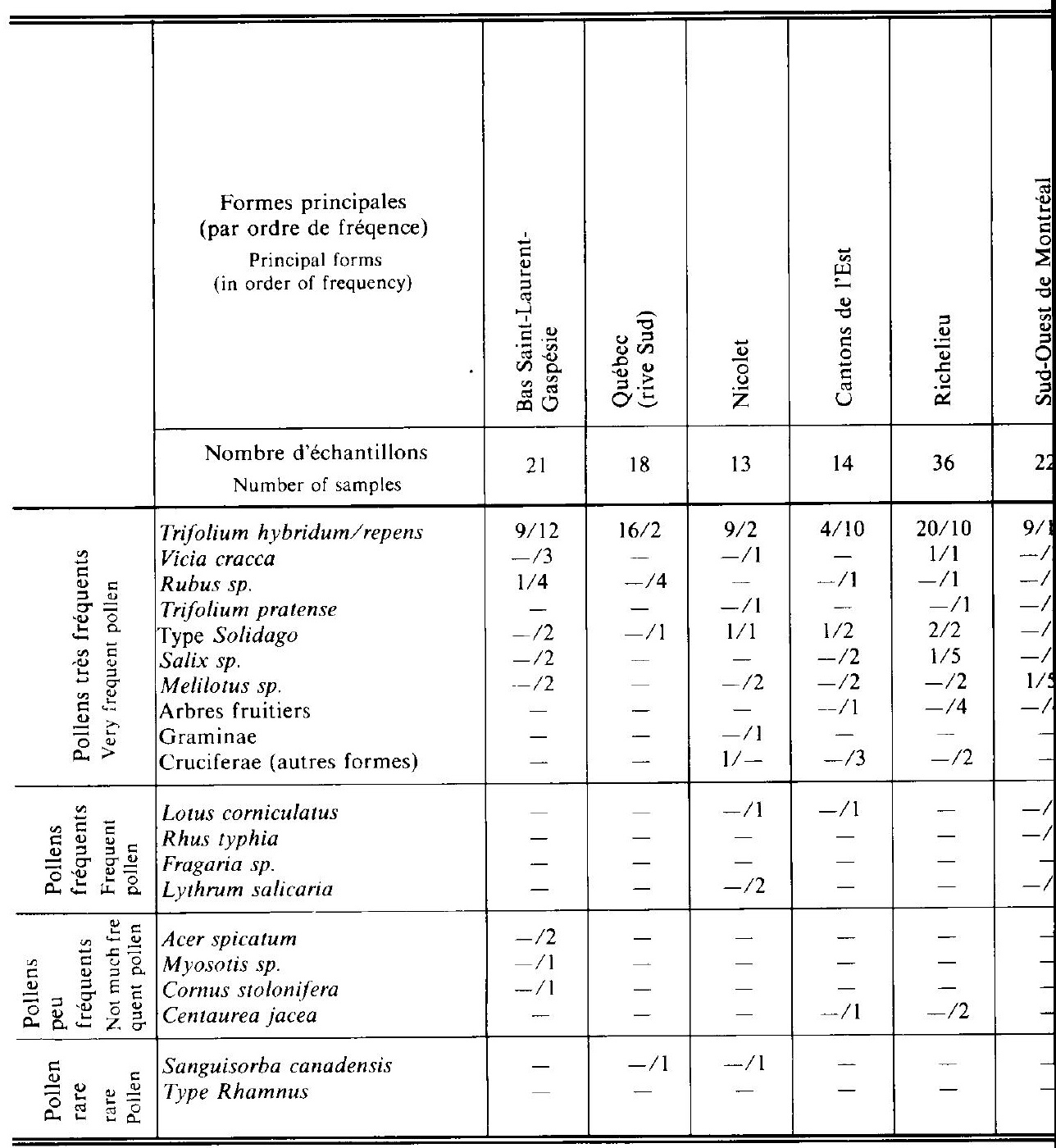


TABL. 6. - Number of dominant pollens (first figure) and the secondary (second figure) in the honey of Quebec and each regional group in order of decreasing frequency

\begin{tabular}{|c|c|c|c|c|c|c|c|c|c|c|}
\hline \multirow[b]{2}{*}{$\begin{array}{l}\frac{n}{\pi} \\
\frac{\tilde{J}}{0} \\
\stackrel{0}{5}\end{array}$} & \multirow[b]{2}{*}{ 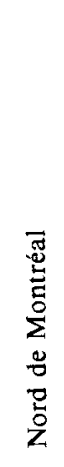 } & \multirow[b]{2}{*}{ 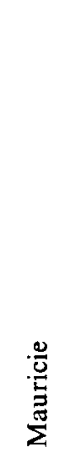 } & \multirow[b]{2}{*}{ 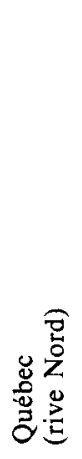 } & \multirow[b]{2}{*}{ 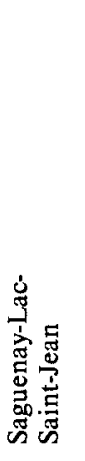 } & \multirow[b]{2}{*}{ ॠ } & \multirow{3}{*}{ 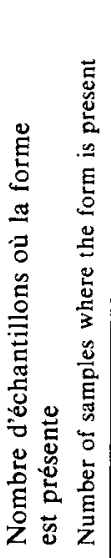 } & \multicolumn{2}{|c|}{$\begin{array}{c}\text { Pollen } \\
\text { dominant } \\
\text { Dominant } \\
\text { pollen }\end{array}$} & \multicolumn{2}{|c|}{$\begin{array}{c}\text { Pollen } \\
\text { d'accom- } \\
\text { pagnement } \\
\text { Secondary } \\
\text { pollen }\end{array}$} \\
\hline & & & & & & & & $\underset{\mathscr{U}}{\mathscr{E}}=$ & 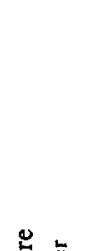 & 苟 $\bar{\Xi}$ \\
\hline 7 & 4 & 6 & 5 & 18 & 164 & & 竞豆 & 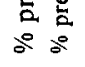 & 豆 & 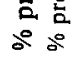 \\
\hline $\begin{array}{l}2 / 5 \\
- \\
- \\
- \\
-/ 1 \\
-1 \\
- \\
- \\
-\end{array}$ & $\begin{array}{l}3 / 1 \\
- \\
- \\
- \\
- \\
- \\
- \\
- \\
-\end{array}$ & $\begin{array}{c}3 / 3 \\
-/ 1 \\
-/ 1 \\
- \\
-/ 1 \\
- \\
- \\
-/ 1 \\
- \\
-/ 1\end{array}$ & $\begin{array}{c}4 / 1 \\
- \\
- \\
- \\
-/ 1 \\
- \\
- \\
- \\
- \\
-\end{array}$ & $\begin{array}{c}13 / 5 \\
-/ 4 \\
- \\
- \\
- \\
-/ 2 \\
- \\
-/ 2 \\
- \\
-\end{array}$ & $\begin{array}{c}92 / 62 \\
1 / 12 \\
1 / 11 \\
-/ 3 \\
4 / 12 \\
1 / 15 \\
1 / 13 \\
-/ 12 \\
-/ 1 \\
1 / 6\end{array}$ & $\begin{array}{r}164 \\
156 \\
144 \\
139 \\
135 \\
132 \\
131 \\
102 \\
93 \\
88\end{array}$ & $\begin{array}{r}92 \\
1 \\
1 \\
- \\
4 \\
1 \\
1 \\
- \\
-1\end{array}$ & $\begin{array}{c}56,10 \\
0,66 \\
0,69 \\
- \\
2,96 \\
0,76 \\
0,76 \\
- \\
- \\
1,14\end{array}$ & $\begin{array}{r}62 \\
12 \\
11 \\
3 \\
12 \\
14 \\
13 \\
12 \\
1 \\
6\end{array}$ & $\begin{array}{r}37,80 \\
7,60 \\
7,64 \\
2,16 \\
8,89 \\
10,60 \\
9,92 \\
11,76 \\
1,07 \\
6,82\end{array}$ \\
\hline $\begin{array}{l}1 / 2 \\
- \\
-\end{array}$ & $\begin{array}{l}- \\
- \\
-\end{array}$ & $\begin{array}{l}- \\
- \\
-1 \\
-\end{array}$ & $\begin{array}{l}- \\
- \\
-\end{array}$ & $\begin{array}{l}- \\
- \\
-\end{array}$ & $\begin{array}{l}1 / 5 \\
-/ 1 \\
-/ 1 \\
-/ 3\end{array}$ & $\begin{array}{l}76 \\
64 \\
58 \\
38\end{array}$ & $\begin{array}{l}1 \\
- \\
- \\
-\end{array}$ & $\begin{array}{l}1,35 \\
- \\
- \\
-\end{array}$ & $\begin{array}{l}7 \\
1 \\
1 \\
1\end{array}$ & $\begin{array}{l}9,46 \\
1,56 \\
1,72 \\
2,63\end{array}$ \\
\hline $\begin{array}{l}- \\
- \\
- \\
-\end{array}$ & $\begin{array}{l}- \\
- \\
-\end{array}$ & $\begin{array}{l}- \\
- \\
-\end{array}$ & $\begin{array}{l}- \\
- \\
- \\
-\end{array}$ & $\begin{array}{l}- \\
-/ 1 \\
- \\
-\end{array}$ & $\begin{array}{l}-/ 2 \\
-/ 2 \\
-/ 1 \\
-/ 3\end{array}$ & $\begin{array}{l}31 \\
26 \\
21 \\
17\end{array}$ & $\begin{array}{l}- \\
- \\
-\end{array}$ & $\begin{array}{l}- \\
- \\
-\end{array}$ & $\begin{array}{l}2 \\
2 \\
1 \\
3\end{array}$ & $\begin{array}{r}6,45 \\
11,54 \\
4,76 \\
17,65\end{array}$ \\
\hline $\begin{array}{l}- \\
-\end{array}$ & $\begin{array}{l}- \\
-\end{array}$ & $\begin{array}{l}- \\
-\end{array}$ & $\begin{array}{c}- \\
-/ 1\end{array}$ & - & $\begin{array}{l}-/ 2 \\
-/ 1\end{array}$ & $\begin{array}{r}16 \\
4\end{array}$ & - & - & $\begin{array}{l}2 \\
1\end{array}$ & $\begin{array}{l}12,5 \\
25\end{array}$ \\
\hline
\end{tabular}


cas des autres formes de cette catégorie de fréquence, dont le pourcentage a augmenté sauf les arbres fruitiers qui paraissent plus réduits.

Le trèfle blanc reste la grande caractéristique des miels du Québec. Il montre le plus grand nombre de cas de dominance et de pollen d'accompagnement et les cas de pollens isolés sont très rares (un seul cas dans cet échantillonnage). Vient ensuite le type Solidago dont les miels dominants sont produits, non seulement, comme nous l'avons déjà cité, dans les comtés longeant le fleuve Saint-Laurent et la rivière des Outaouais (Feller-Demalsy et LAmontagne, 1979), mais aussi à proximité des rivières importantes telle la rivière Richelieu. Pour Vicia cracca, un cas de pollen dominant a été observé dans la région de Richelieu (comté de Chambly) et les cas de pollens d'accompagnement ne sont pas rares $(5,16 \%$ des échantillons pour les 2 études réunies). Cette abondance de pollens de cette forme paraît caractéristique puisqu'elle est réputée ne se trouver dans les miels qu'à l'état de pollen isolé (Maurizio et Louveaux, 1965). Trifolium pratense et Salix pour lesquels la fréquence moyenne de formes ne s'est que peu modifiée au cours des 2 études, y montrent aussi des caractéristiques locales semblables. Les cas de pollen dominant de Trifolium pratense n'ont pas été retrouvés, ce qui confirme bien la rareté de ce type de miel (Maurizio et Louveaux, 1965, Louveaux, 1966) mais quelques cas de pollens d'accompagnement $(2,44 \%)$. Cette étude a confirmé sa fréquence plus élevée dans les Cantons de l'Est et plus basse dans l'Outaouais. Les formes de Salix plus abondantes dans les miels du Sud, du Sud-Ouest (surtout dans la région du Richelieu avec un cas de pollen dominant et quatre de pollen d'accompagnement) et de l'Ouest restent toujours caractéristiques de l'Outaouais où se rencontre la plus grande fréquence. Les deux études réunies montrent également une fréquence élevée au SaguenayLac-Saint-Jean, mais basse dans la région de Québec rive Nord. La fréquence de pollens d'arbres fruitiers dans le miel ne paraît pas toujours en relation avec l'importance des vergers au Québec. Il est vrai que ce type de pollen inclut également des pollens de plantes spontannées telles des Amelanchiers dont les espèces sont répandues dans toute la province. Cependant à la présence de vergers importants correspondraient plutôt des cas de pollen d'accompagnement comme on peut l'observer dans la région du Richelieu, du Sud-Ouest et du Nord de Montréal où le nombre de pommier est le plus élevé (LABRECQUE et THIBEAULT, 1978). Quatre formes présentent une fréquence 2 à 3 fois plus élevée dans les miels de la deuxième étude. Il s'agit de Rubus, Melilotus, le type Taraxacum et les Crucifères (autres formes). Il est à noter que ces plantes ne sont pas rares au Québec et que la fréquence de leurs formes dans les miels n'a rien d'étonnant. En outre le framboisier (Rubus idaeus), qui est spontané est aussi cultivé dans presque toutes les régions du Québec. Un cas de dominance a été trouvé dans le Bas Saint-Laurent-Gaspérie (comté de Rivière-du-Loup), et les cas de pollen d'accompagnement et isolés abondants y sont fréquents. Selon Maurizio et Louveaux (1965), les miels de Rubus sont rares, mais déjà signalés au Canada. Suivant PELLET (1977) ils sont produits lorsque la plante est abondante, qu'elle soit spontanée ou cultivée. Le cas des Crucifères est plus 
complexe parce que se rapportant à des genres divers. Une fréquence plus élevée accompagnée d'une certaine abondance se remarque au Sud du Fleuve SaintLaurent. Ces cas ne paraissent pas en relation avec la culture du Colza, d'ailleurs peu pratiquée au Québec, leurs sites en différant (LABRECQUE et THIBAULT, 1978), mais paraissent plus attribuables à des adventices de cultures telles Brassica campestris ou à d'autres plantes spontanées ou subspontanées telles Barbarea vulgaris très abondante au printemps dans les fossés et au bord des chemins (Frère MARIE-VICTORIN, 1964; RoussEAU, 1968). Toutes deux sont des plantes nectarifères et pollinifères.

Les formes indicatrices géographiques des régions citées dans la première étude ont été retrouvées, qu'elles correspondent ou non à une distribution limitée de la plante. La présente étude a permis d'apporter des précisions pour plusieurs d'entre elles. Ainsi les formes de Lythrum salicaria (plante de milieu humide) identifiées comme caractéristiques des miels de la rive Nord du Saint-Laurent, le sont également des miels de la rive Sud, où elles atteignent la plus grande fréquence dans le Nicolet en plus d'une certaine abondance ( 2 cas de pollen d'accompagnement) et aussi le long des rivières importantes (la rivière des Outaouais et la rivière Richelieu). Sanguisorba canadensis caractéristique des territoires riverains du fleuve SaintLaurent de l'Est du Québec chevauche avec la précédente dans les régions de Québec, de Nicolet (comté de Nicolet) et, Comme en témoigne la précédente étude, de la Mauricie (comté de Champlain). Les pollens de Lotus corniculatus et de Cornus stolonifera sont respectivement plus fréquents et plus abondants dans les miels de l'Ouest et de l'Est de la province bien que présents dans toutes les régions. Toutefois les formes de Lotus corniculatus paraissent extrêmement rares dans les miels du Nord du territoire étudié (Saguenay-Lac-Saint-Jean, 1 cas sur 36 échantillons, comté de Chicoutimi - voir étude des miels de 1975). Les formes de Polygonum fagopyrum paraissent bien caractéristiques des régions où la culture en est plus intensive (Nord de Montréal, Sud-Ouest de Montréal, Richelieu, Nicolet et Mauricie - données des deux études). La forme de Centaurea jacea/nigra est caractéristique de miels de 3 comtés voisins situés au sud de la province : le Missisquoi, Brome et le Sud du Comté de Rouville. Dans ceux-ci, il s'agit manifestement de Centaurea jacea dont nous avons pu vérifier sur place la présence et l'abondance de cette plante limitée à ces territoires. Des cas de pollen d'accompagnement de cette forme avaient aussi été observés dans l'étude des miels de 1975 dans le comté de Missisquoi. N'étant signalée que rarement (MAUrizio et LouveAuX, 1965), cette abondance associée à la fréquence constitue une caractéristique de ces comtés. Pour les miels du Bas Saint-Laurent-Gaspésie et probablement de toutes les régions de même latitude, il s'agit manifestement de Centaurea nigra. Myosotis sp. bien qu'étant une plante largement répandue (Frère MARIE-VICTORIN, 1964) et rattachée aux milieux humides et frais voit ses formes cantonnées surtout dans les miels de l'Est de la province (Bas Saint-Laurent et Saguenay-Lac-Saint-Jean). Il en est apparemment de même des formes d'Impatiens $s p$. qui bien que irrégulièrement retrouvées dans les miels paraît surtout rattachées aux miels des régions de Nicolet et des Cantons de l'Est. 
Parmi les formes de pollen appartenant à des plantes cultivées, deux marquent les miels de toutes les régions: Medicago et Fragaria (sauf le Nord-Ouest québécois pour Fragaria). Medicago présente une fréquence plus élevée dans les miels du Sud-Ouest de Montréal (59,09 \%) et du Bas Saint-Laurent-Gaspésie (57,14\%) bien qu'il soit cultivé dans toutes les régions du Québec. Les formes de Fragaria atteignent les fréquences les plus élevées en Mauricie ( $40 \%$ en 1975 et $75 \%$ en 1978), bien que les surfaces cultivées n'y soient pas les plus importantes mais plutôt au Nord de Montréal et dans la région de Québec. L'apport provenant de fraisiers spontanés (Fragaria virginiana DUCHESNE) abondants dans les pâturages et les lieux incultes (RouSSEAU, 1974) n'est certes pas à négliger.

Plusieurs formes nouvelles ont pu être retracées dont les plus importantes sont : Rhus typhina et Acer spicatum. La première est caractéristique des miels du SudOuest de la province (surtout des comtés de Soulanges, de Beauharnois, de Brome et du Missisquoi) mais peut être retrouvée dans les miels d'autres régions à l'exclusion de l'Est et du Nord du territoire étudié (Bas-Saint-Laurent-Gaspésie et du Saguenay-Lac-Saint-Jean). Cette caractéristique est étayée par l'abondance de la plante dans le Sud-Ouest de la province (observations personnelles) surtout dans les milieux secs et rocheux (Frère MARIE-VICTORIN 1964). Rhus typhina est une espèce très nectarifère et très pollinifère dont la floraison inaugure la grande miellée (vers la mi-juin dans la région de Montréal) (Frère MARIE-VICTORIN 1964). Acer spicatum est caractéristique des miels de l'Est de la province et principalement du Bas Saint-Laurent-Gaspésie où la fréquence est élevée $(90,40 \%)$ et où 2 cas de pollen d'accompagnement ont été relevés (comté de Matane). Il est présent dans les miels de la rive Nord du Saint-Laurent mais sur la rive Sud parait absent de plusieurs comtés (du Nicolet, du Richelieu et des cantons de l'Est). Il s'agit d'un érable répandu dans tout le Québec (Rousseau 1979), très nectarifère, fleurissant au mois de juin, mais dont les abeilles ne récoltent le nectar qu'en période de disette (PELLET 1977). Les formes de type Rhamnus sont extrêmement rares (observées dans 4 miels) mais donnent lieu à un cas de pollen d'accompagnement dans le comté de Québec (région de Québec rive Nord. Il est à noter que le Rhamnus cathartica en est la seule espèce abondante au Québec mais seulement autour de certaines villes (Frère MARIEVICTORIN 1964).

Parmi les formes caractéristiques par leur seule fréquence se rencontrent des plantes spontanées ou subspontanées souvent adventices de cultures. Il s'agit de : Renonculacées, Ombellifères, type Chrysanthemum, Rumex acetosella (Tableau 5) et Arctium minus (Bas-Saint-Laurent-Gaspésie, Saguenay-Lac-Saint-Jean).

Les autres formes de pollen paraissent sporadiques bien que certaines pourraient être intéressantes au point de vue géographique : Ambrosia centre-ouest de la province (fréquence relativement élevée en Mauricie, $40 \%$ dans la première étude et $33,33 \%$ dans la deuxième), Caryophyllacées/Chénopodiacées, sans doute en relation avec certaines cultures (adventices), Alnus au centre et à l'est de la province, 
Echium et Tilia americana dans l'Ouest du territoire (en fait caractéristiques des miels de l'Ontario - Louveaux 1966), Syringa vulgaris au Sud-Ouest de Montréal bien que la plante soit présente partout.

La principale modification apportée à la composition des miels durant ces quatre années sont la forte augmentation de fréquence déjà signalée de quelques formes de pollen. Certaines peuvent être rattachées à l'intensification de cultures comme pour le fraisier et le framboisier (LABRECQUE et THIBAULT 1978), d'autres plutôt à des séquelles de cultures comme les cas de résistance aux herbicides sélectifs qui ont été signalés pour la moutarde des oiseaux (Brassica campestris) le Chénopode blanc (Chenopodium album) et la petite oseille (Rumex acetosella) (EMOND 1981). Même la végétation spontanée, qui paraît soumise à des changements moins brusques, peut par le jeu des conditions climatiques voir modifier sa production de nectar. Ainsi, la fréquence et l'abondance des formes de Melilotus dans les miels de 1978 pourrait être en relation avec un temps plus sec et plus ensoleillé en juillet de cette année qu'en 1975 (service de la météorologie 1975 et 1978), cette plante produisant plus de nectar dans les régions à climat chaud et sec (Pellet 1977). Par ailleurs, il ne serait pas impossible qu'il puisse exister une relation entre cet été favorable et l'augmentation du nombre de formes de pollen dans les miels de cette année.

Cette étude permet de constater que le nombre de formes d'importance majeure pour les miels au Québec est plus élevé qu'il n'apparaissait à première vue. L'importance de Vicia cracca permet de l'ajouter aux formes déjà énoncées qui s'élèvent alors au nombre de 5 : Trifolium, Vicia cracca, le type Solidago, les arbres fruitiers et Salix. Trois autres formes paraissent en outre d'importance majeure pour la rive Sud du Saint-Laurent : Rubus, Melilotus (par les étés chauds et secs) et les Crucifères. Les caractéristiques régionales ont été confirmées. Deux nouveaux indicateurs géographiques ont été décelés: Rhus typhina pour l'ouest et le sud-ouest de la province et Acer spicatum pour l'Est. Ce nouvel échantillonnage a par ailleurs confirmé la rareté des formes de Tilia americana, d'Epilobium (Onagraceae) et de Vaccinium bien que SLADEN (Pellet 1977) les signale d'importance majeure au Québec. Quant à Acer, cet échantillonnage a permis de déceler Acer rubrum et Acer saccharum espèces à floraison printanière dont les formes sont pour la plupart du temps isolées et isolées importantes et Acer spicatum à floraison moins précoce (juin) qui prend quelqu'importance dans les miels du Bas Saint-Laurent, Gaspésie.

Si cet étude complémentaire a permis de percevoir une évolution de la composition des miels soumise, d'une part, aux fluctuations de certaines cultures, d'autre part, aux variations de climat, les caractères généraux sont restés inchangés, peu de choses sont infirmées, elles sont plutôt précisées par l'apport de données nouvelles. Cette étude a permis ainsi d'établir avec certitude des caractéristiques géographiques qui ne paraissent pas ou très peu fluctuer avec le temps, tant pour le Québec que pour chacune de ses parties. Puisque l'origine de l'échantillonnage est différent à $80 \%$, il 


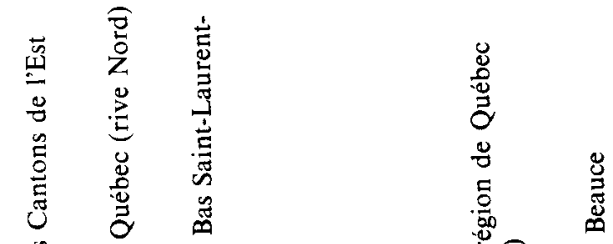

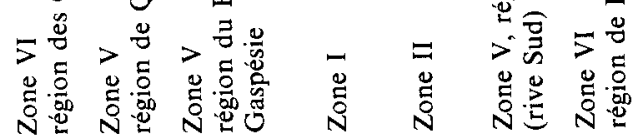

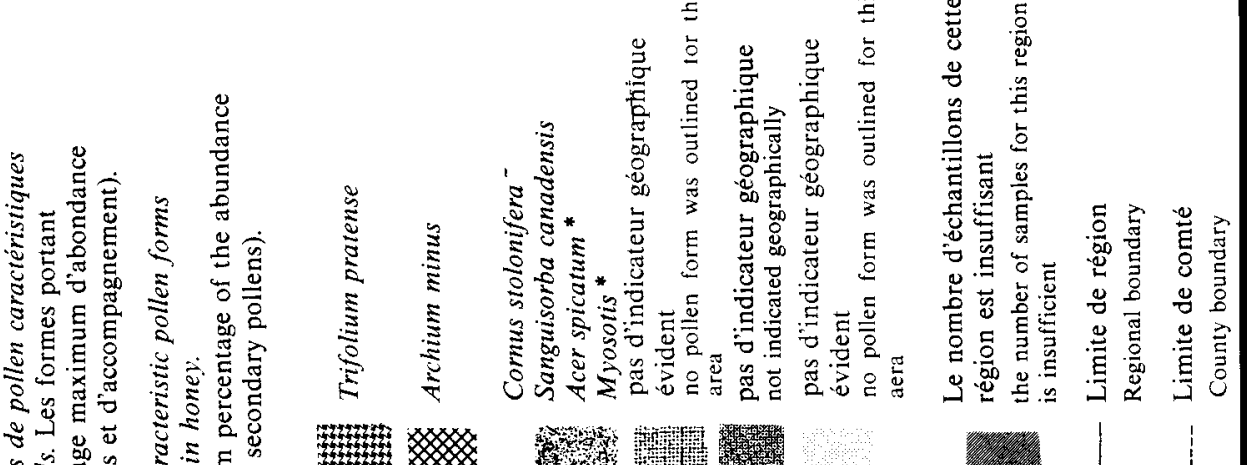

ช્山.

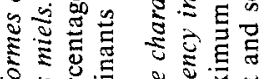

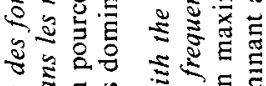

๖ 马

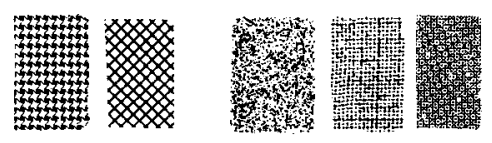

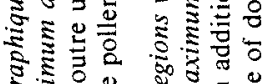

क力

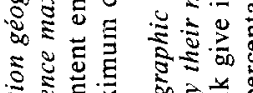

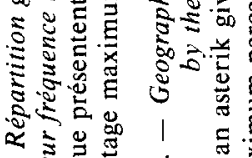

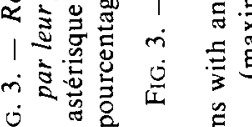

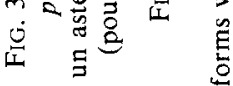

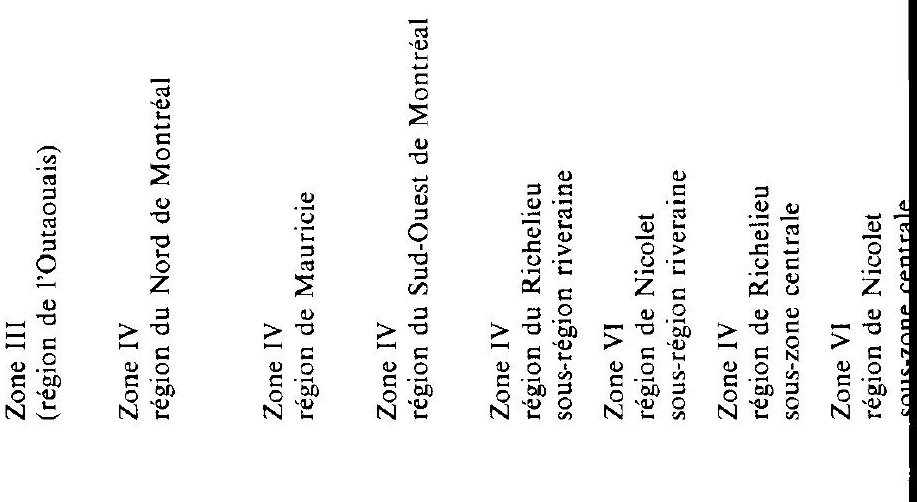

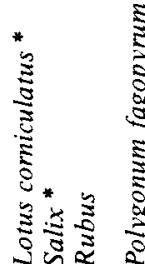

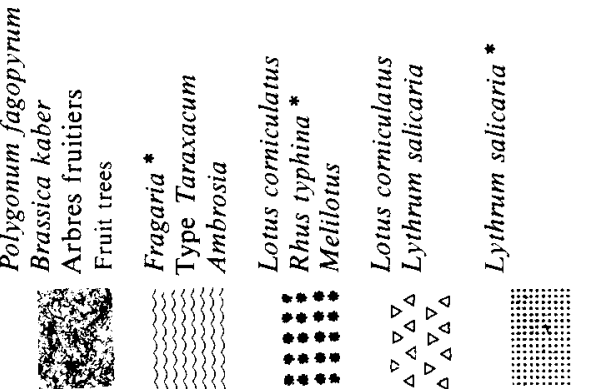
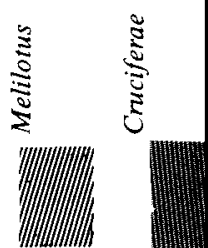


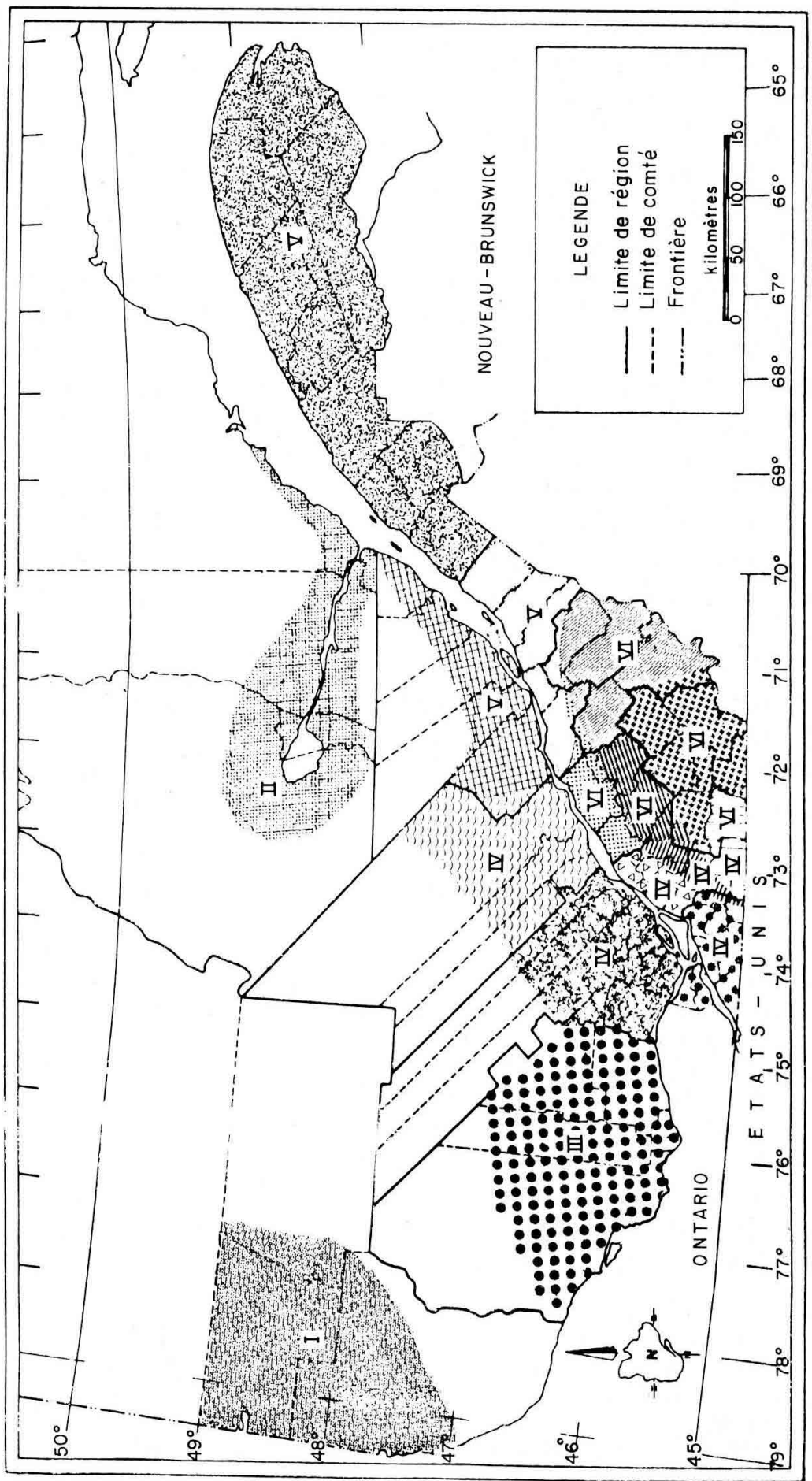


est intéressant de considérer l'ensemble des deux études, ce qui donne 368 spécimens de miel et un échantillonnage très valable pour chacune des régions en particulier (sauf la région de Beauce), ce qui n'est pas nécessairement le cas pour chaque étude séparée (1).

Les zones apicoles telles qu'elles ont été délimitées dans la publication de 1979 (Feller-Demalsy et Lamontagne) sont maintenues dans l'ensemble, mais l'échantillonnage assez important pour certaines d'entre elles permet de mieux préciser les caractéristiques de chacune voire dans certains cas de délimiter des sous-zones (figure 3). A la division plus ou moins verticale correspondant aux zones s'ajoute une subdivision plus ou moins horizontale influencée principalement par la présence du fleuve important qu'est le Saint-Laurent mais aussi par certaines caractéristiques géologiques. Cette subdivision de certaines zones déjà pressentie précédemment et que le nouvel échantillonnage a permis de mieux préciser, s'effectue en quatre sous-zones : la rive Nord et la rive Sud, sous-zone riveraine, sous-zone centrale et sous-zone méridionale. Les zones impliquées sont celles du Centre-Ouest, du Sud-Ouest et celle du Sud-Est. Les limites restent encore imprécises en ce qui concerne la Beauce dont l'échantillonnage de miel est trop réduit (tableau 7 et figure 3). Les miels de la rive Nord se caractérisent par une fréquence plus réduite de Salix de Crucifères, de Melilotus, de Rubus, de Graminées, de Lotus corniculatus, d'Acer et de Rhus typhina. Par contre Polygonum fagopyrum et Fragaria y sont plus fréquents. Quant à la rive Sud, les miels de la sous-zone riveraine montrent une plus grande fréquence de Lythrum salicaria et de Lotus corniculatus, dans la sous-zone méridionale, de Rhus typhina (dont la fréquence diminue dans les miels vers l'Est) et pour les comtés de Missisquoi et de Brome à l'extrême Sud de la province, de Centaurea jacea. Dans la sous-zone centrale de transition, les caractères des souszones précédentes se rencontrent mais de façon atténuée. Les pollens de Melilotus y paraissent plus importants. Dans la région du sud-ouest de Montréal, cette subdivision est moins claire, mais Lythrum salicaria est absent des miels du sud de la région et Lotus corniculatus y est rare, tandis que dans les comtés longeant le fleuve, tous les caractères de l'Ouest de la province sont réunis : présence fréquente et en quantité appréciable de Lythrum salicaria, Rhus typhina, Lotus corniculatus et Polygonum fagopyrum. C'est cependant en Outaouais que Lotus corniculatus est le plus fréquent et le plus abondant. Cette forme de pollen ainsi que celle de Rhus typhina paraissent toutefois absentes de miels provenant du comté de Labelle situé au nord de la région. Des formes de pollent distinctes caractérisent les miels de l'Est du Québec. Dans le Bas-Saint-Laurent-Gaspésie, il s'agit de Cornus stolonifera

(1) L'examen des 2 études conjointes se justifie aussi par le fait que dans chacune, certains échantillonnages de région sont insuffisants pour obtenir un bon aperçu de la flore impliquée dans leurs miels. (Des courbes de type Guinochet ont été effectuées pour s'en assurer.) (LoUvEauX et Vergeron 1964) et que la déficience d'échantillonnage d'une étude a pu être compensée par l'échantillonnage valable de l'autre. C'est le cas par exemple des régions de Québec rive nord et du nord de Montréal de cette étude et de la région de Nicolet du premier travail. 
TABL. 7. - Formes de pollens caractéristiques des miels des différentes zones et sous-zones apicoles abstraction faite de Trifolium hybridum/repens, Viccia cracca et type Solidago.

sont mentionnés que pour la fréquence et le pourcentage d'abondance maxima pour le Québec. Dans celui-ci sont inclus tous les cas de pollen dominant

ou d'accompagnement pour les miels d'un territoire donné.

Les autres formes de pollen citées ne comprennent que les plus caractéristiques.

TABL. 7. - Forms of pollen characteristic of honey of the different beekeeping zones and sub-zones

For the other forms, these are in the high frequency and the percentage of maximum abundance for Quebec.

In the latter, will be included all the cases of dominan

The other forms of pollen only refer to the most characteristic.

\begin{tabular}{|c|c|c|c|c|c|c|}
\hline Zones et régions & $\begin{array}{l}\text { Fréquence } \\
\text { maximum } \\
\text { (pour le Québec) } \\
\\
\text { Maximum } \\
\text { frequency } \\
\text { (for Quebec) }\end{array}$ & $\begin{array}{c}\text { Pourcentage } \\
\text { maximum } \\
\text { d'abondance } \\
\text { (pour le Québec) } \\
\text { Maximum } \\
\text { percentage } \\
\text { of abondance } \\
\text { (for Quebec) }\end{array}$ & Fréquents & Peu fréquents & $\begin{array}{l}\text { Rares } \\
\text { Rarely }\end{array}$ & $\begin{array}{l}\text { Absence } \\
\begin{array}{l}\text { Not } \\
\text { detected }\end{array}\end{array}$ \\
\hline \multicolumn{7}{|l|}{ I - Nord-Ouest } \\
\hline $\begin{array}{l}\text { Nord-Ouest } \\
\text { québécois }\end{array}$ & - & - & $\begin{array}{l}\text { Lotus corniculatus } \\
\text { (et abondance rela- } \\
\text { tive) }\end{array}$ & Cornus stonolifera & & $\begin{array}{l}\text { De formes } \\
\text { caractéristiques }\end{array}$ \\
\hline \multicolumn{7}{|l|}{ II - Centre-Nord } \\
\hline $\begin{array}{l}\text { Saguenay-Lac- } \\
\text { Saint-Jean }\end{array}$ & - & Vicia cracca & & Arctium minus & $\begin{array}{l}\text { Sanguisorba cana- } \\
\text { densis } \\
\text { Lotus corniculatus } \\
\text { Polygonium fagopy- } \\
\text { rum } \\
\text { Lythrum salicaria } \\
\text { Cornus stolonifera }\end{array}$ & Rhus typhina \\
\hline \multicolumn{7}{|l|}{ III - Ouest } \\
\hline Outaouais & \begin{tabular}{|l} 
Salix \\
Rubus \\
Lotus corniculatus
\end{tabular} & $\begin{array}{l}\text { Salix } \\
\text { Lotus corniculatus }\end{array}$ & \begin{tabular}{|l|} 
Lythrum salicaria \\
Polygonum fagopy- \\
rum \\
Rhus yphina \\
Echium
\end{tabular} & - & $\begin{array}{l}\text { Cornus stolonifera } \\
\text { Arctium minus } \\
\text { Centaurea jacea/ni- } \\
\text { gra }\end{array}$ & \\
\hline \multicolumn{7}{|l|}{$\begin{array}{l}\text { IV - Centre Ouest- } \\
\text { Sud-Est }\end{array}$} \\
\hline \multicolumn{7}{|l|}{ Rive Nord : } \\
\hline de Montréal & $\begin{array}{l}\text { arbres fruitiers } \\
\text { Brassica kaber } \\
\text { Polygonum fagopy- } \\
\text { rum }\end{array}$ & & & $\begin{array}{l}\text { Lotus corniculatus } \\
\text { Rhus typhina } \\
\text { Echium }\end{array}$ & $\begin{array}{l}\text { Cornus stolonifera } \\
\text { Arctium minus }\end{array}$ & $\begin{array}{l}\text { Sanguisorba } \\
\text { canadensis } \\
\text { Centaurea jacea/ } \\
\text { nigra }\end{array}$ \\
\hline - Mauricie & $\begin{array}{l}\text { Fragaria } \\
\text { Type Taraxacum } \\
\text { Ambrosia }\end{array}$ & Fragaria & & \begin{tabular}{|l|} 
Lotus corniculatus \\
Rhus typhina \\
Cornus stolonifera \\
Sanguisorba cana- \\
densis \\
Arctium minus
\end{tabular} & & $\begin{array}{l}\text { Centaurea jacea/ } \\
\text { nigra }\end{array}$ \\
\hline \multicolumn{7}{|l|}{$\begin{array}{l}\text { Rive Sud, sous- } \\
\text { rive riveraine : }\end{array}$} \\
\hline - Sud-Ouest de & Melilotus & Melilotus & \begin{tabular}{|l|}
$\begin{array}{l}\text { Polygonum fagopy- } \\
\text { rum }\end{array}$ \\
\end{tabular} & Cornus stolonifera & $\begin{array}{l}\text { Centaurea jacea/ni- } \\
\text { gra }\end{array}$ & $\begin{array}{l}\text { Sanguisorba cana- } \\
\text { densis }\end{array}$ \\
\hline Montréal & $\begin{array}{l}\text { Lotus corniculatus } \\
\text { Rhus typhina }\end{array}$ & $\begin{array}{l}\text { Trifolium pratense } \\
\text { Rhus typhina }\end{array}$ & Lythrum salicaria & Echium & & \\
\hline \multirow[t]{2}{*}{$\begin{array}{l}\text { - Richelieu } \\
\text { (nord) }\end{array}$} & Lythrum salicaria & & & Rhus typhina & Cornus stolonifera & $\begin{array}{l}\text { Sanguisorba cana- } \\
\text { densis }\end{array}$ \\
\hline & Lotus corniculatus & & & $\begin{array}{l}\begin{array}{l}\text { Polygonum fagopy- } \\
\text { rum }\end{array} \\
\end{array}$ & & $\begin{array}{l}\text { Centaurea jacea/ni- } \\
\text { gra }\end{array}$ \\
\hline $\begin{array}{l}\text { - Nicolet (Com- } \\
\text { tés de }\end{array}$ & Lythrum salicaria & Lythrum salicaria & & $\begin{array}{l}\begin{array}{l}\text { Sanguisorba } \\
\text { densis }\end{array} \\
\text { cana- }\end{array}$ & & $\begin{array}{l}\text { Centaurea jacea/ni- } \\
\text { gra }\end{array}$ \\
\hline $\begin{array}{l}\text { Yamaska et de } \\
\text { Nicolet) }\end{array}$ & & & & $\begin{array}{l}\text { Polygonum fagopy- } \\
\text { rum }\end{array}$ & & Arctium minus \\
\hline \multicolumn{7}{|l|}{$\begin{array}{l}\text { Rive Sud, sous- } \\
\text { zone centrale : }\end{array}$} \\
\hline $\begin{array}{l}\text { - Richelieu } \\
\text { (centre) }\end{array}$ & Melilotus & & $\begin{array}{l}\text { Rhus typhina } \\
\text { Polygonum fagopy- } \\
\text { rum }\end{array}$ & & $\begin{array}{l}\text { Arctium minus } \\
\text { Centaurea jacea }\end{array}$ & $\begin{array}{l}\text { Cornus stolonifera } \\
\text { Sanguisorba cana- } \\
\text { densis }\end{array}$ \\
\hline $\begin{array}{l}- \text { Nicolet (com- } \\
\text { tés de }\end{array}$ & Cruciferes & Graminées & \begin{tabular}{|l|} 
Polygonum fagopy- \\
rum
\end{tabular} & Rhus typhina & Alnus & $\begin{array}{l}\text { Centaurea jacea/ni- } \\
\text { gra }\end{array}$ \\
\hline $\begin{array}{l}\text { Drummond et } \\
\text { d'Arthabasca) }\end{array}$ & & & Lythrum salicaria & $\begin{array}{l}\text { Echium } \\
\text { Lotus corniculatus }\end{array}$ & Cornus stolonifera & Arctium minus \\
\hline $\begin{array}{l}- \text { Beauce } \\
\text { (2 miels) }\end{array}$ & & & $\begin{array}{l}\text { échantillonna } \\
\text { abondance relative de }\end{array}$ & $\begin{array}{l}\text { ge trop faible } \\
\text { Salix et de Melilons }\end{array}$ & & \\
\hline \multicolumn{7}{|l|}{$\begin{array}{l}\text { Rive Sud, sous- } \\
\text { zone méridionale }\end{array}$} \\
\hline $\begin{array}{l}\text { - Richelieu } \\
\text { (comté de Missis- }\end{array}$ & Centaurea jacea & Centaurea jacea & $\begin{array}{l}\text { Polygonum fagopy- } \\
\text { rum }\end{array}$ & Lythrum salicaria & Cornus stolonifera & $\begin{array}{l}\text { Sanguisorba cana- } \\
\text { densis }\end{array}$ \\
\hline & $\begin{array}{l}\text { Rubus } \\
\text { Rhus typhina }\end{array}$ & & Lotus corniculatus & & $\begin{array}{l}\text { Arctium minus } \\
\text { Echium }\end{array}$ & \\
\hline $\begin{array}{l}\text { - Cantons de } \\
\text { l'Est }\end{array}$ & Rhus typhina & & Lotus corniculatus & $\begin{array}{l}\text { Polygonum fagopy- } \\
\text { rum }\end{array}$ & & Lythrum salicaria \\
\hline $\begin{array}{c}\text { (comté de } \\
\text { Brome) (5 miels) }\end{array}$ & Centaurea jacea & & & & & $\begin{array}{l}\text { Cornus stolonifera } \\
\text { Sanuisorba cana- } \\
\text { densis } \\
\text { Arctum minus. }\end{array}$ \\
\hline $\begin{array}{l}\text { - Cantons de } \\
\text { l'Est }\end{array}$ & Trifolium pratense & Impatiens capensis & Rhus typhina & Lotus corniculatus & $\begin{array}{l}\text { Polygonum fagopy- } \\
\text { rum }\end{array}$ & $\begin{array}{l}\text { Sanguisorba cana- } \\
\text { densis }\end{array}$ \\
\hline $\begin{array}{l}\text { (moins comté de } \\
\text { Brome) }\end{array}$ & & Crucifères & & & $\begin{array}{l}\text { Cornus stolonifera } \\
\text { Lythrum salicaria } \\
\text { Centaurea jacea/ni- } \\
\text { gra }\end{array}$ & Arctium minus \\
\hline \multicolumn{7}{|l|}{ V - Centre-Est et Est } \\
\hline rive & Arctium minus & $\begin{array}{l}\text { Arbres fruitiers } \\
\text { Type Rhammus }\end{array}$ & $\begin{array}{l}\text { Lotus corniculatus } \\
\text { Sanguisorba cana- } \\
\text { densis } \\
\text { Lythrum salicario } \\
\text { Polygonum fagopy- } \\
\text { rum }\end{array}$ & $\begin{array}{l}\text { Cornus stolonifera } \\
\text { Alnus }\end{array}$ & $\begin{array}{l}\text { Rhus typhina } \\
\text { Centaurea nigra } \\
\text { Echium }\end{array}$ & \\
\hline $\begin{array}{l}\text { - Québec } \\
\text { rive Sud }\end{array}$ & & \begin{tabular}{|l|} 
Rubus \\
Sanguisorba cana- \\
densis
\end{tabular} & Lotus corniculatus & \begin{tabular}{|l} 
Lythrum salicaria \\
Cornus stolonifera \\
Sanguisorba cana- \\
densis \\
Polygonum fagopy- \\
rum \\
Arctium minus \\
Alnus
\end{tabular} & $\begin{array}{l}\text { Rhus typhina } \\
\text { Centaurea nigra }\end{array}$ & \\
\hline
\end{tabular}


For the other forms, these are in the high frequency and the percentage of maximum abundance for
Quebec.

In the latter, will be included all the cases of dominan

or secondary pollen for the honey of a given territory.

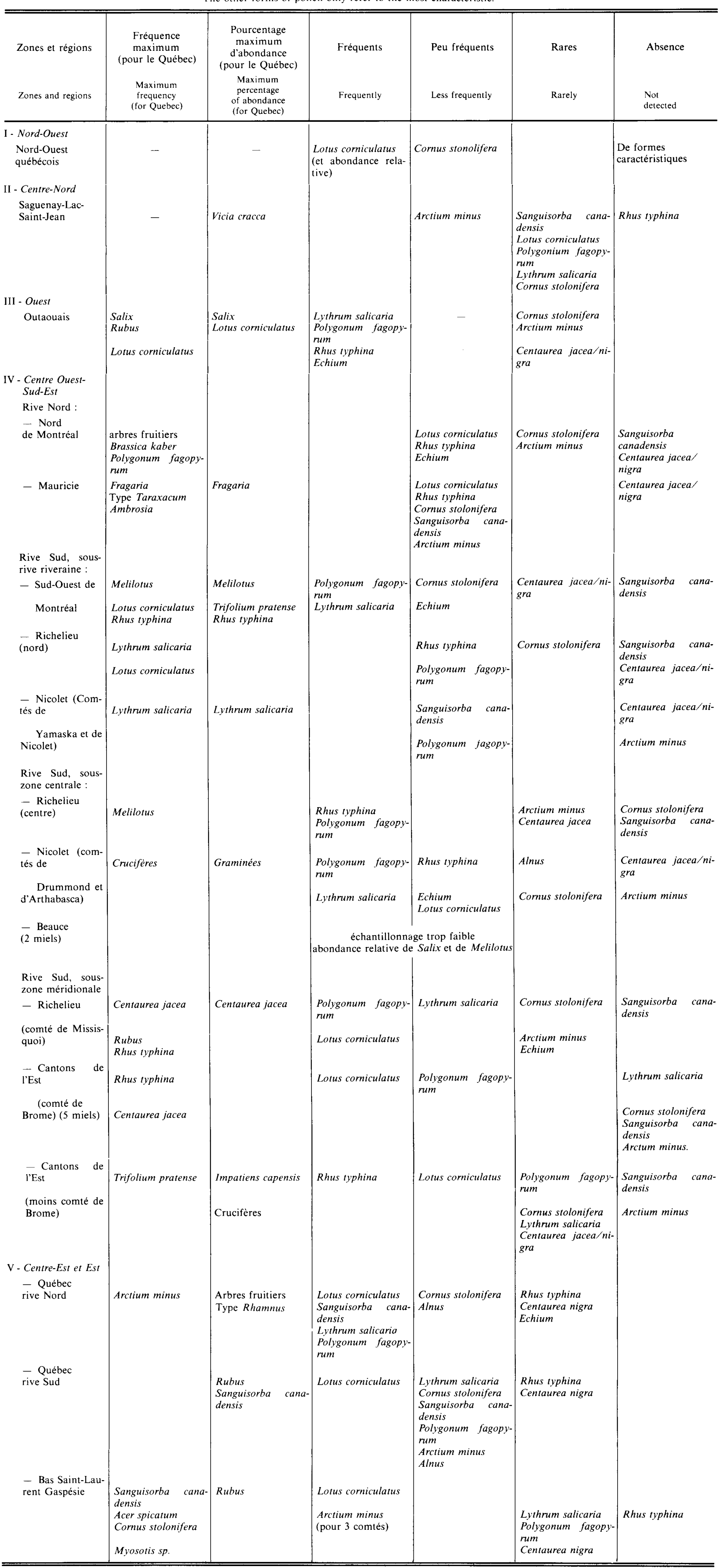


$(34,78 \%)$ (surtout les comtés de Matane et de Rimouski), de Sanguisorba canadensis $(43,48 \%)$ (surtout le comté de Rivière-du-Loup), de Graminées, d'Acer spicatum $(90,48 \%)$, de Myosotis $(26,09 \%)$ (surtout les comtés de Rivière-du-Loup et de Kamouraska) et de Arctium minus (comtés de Rimouski, de Rivière-du-Loup et de Kamouraska). Les régions de Nicolet (zone VI Sud-Est) et de Québec (rive Sud) (zone V Centre-Est) constituent la zone de transition entre l'est et l'ouest pour la rive Sud. Si les formes caractéristiques de l'Ouest sont encore présentes sporadiquement dans les miels (Rhus typhina dans les miels des comtés de Drummond et d'Arthabask ‘), se devinent déjà des caractéristiques de l'Est dans les miels du comté de Nicolet (présence de pollens de Sanguisorba canadensis). Les pollens de Lythrum salicaria et des Crucifères y ont leur fréquence la plus élevée. Les deux miels de la région de Beauce (comté de Dorchester et de Mégantic) (étude publiée en 1979, FELLER-DemalsY et LAMONTAGNE) sont insuffisants pour caractériser cette région. Toutefois deux points peuvent être retenus : la présence à l'état de pollen isolé important de Salix et de Melilotus. La région de Québec (rive Sud) continue la transition vers l'Est. Les pollens caractéristiques des miels de l'Est et de l'Ouest de la province y sont toutefois rares. Sur la rive Nord, la transition entre l'ouest et l'est commence en Mauricie (présence de Sanguisorba canadensis dans les miels du comté de Champlain) et se prolonge dans la région de Québec (rive Nord) où les formes caractéristiques de l'Ouest sont encore présentes dans les miels (Rhus typhina) tandis que celles de l'Est y prennent déjà de l'importance (Sanguisorba canadensis et Arctium minus). Dans les miels des deux zones situées au nord de la province, ces pollens particuliers sont absents ou leur présence est exceptionnelle. Mais dans le Nord-Ouest québécois (zone 1), les miels se rapprochent de ceux de l'Outaouais par une abondance relative de Salix et de Lotus corniculatus, et dans la zone Centre-Nord (zone II), la fréquence et l'abondance de Vicia cracca est la plús élevée au Québec.

La considération de l'ensemble des deux échantillonnages montre en outre que les moyennes de nombre de formes de pollen sous la moyenne au Québec se rencontrent dans les miels des régions les plus septentrionales, ce qui avait déjà été observé, mais aussi dans ceux des régions dont le pourcentage de terres cultivées est le plus élevé ( 80 à $95 \%$ des terres agricoles) (Service des études économiques 1978) (voir figure 4). Il semble bien que ce nombre soit ici en relation à la fois avec des conditions climatiques et l'activité humaine. Ce nombre augmente brusquement à partir du comté de l'Islet et dans le Bas-Saint-Laurent-Gaspésie. Il ne semble pas que les facteurs précités puissent être la cause d'un changement aussi brusque à cet endroit, mais il est possible qu'un ensemble de facteurs dont l'environnement marin. y soient en jeu. C'est dans cette région que se rencontre aussi la plus grande variété de pollens dominants et d'accompagnement autre que le trèfle. Certains lui sont même propres (Cornus stolonifera et Acer spicatum).

Les miels du Québec possèdent globalement les caractéristiques décrites par Louveaux (1966) pour les miels de l'est du Canada. Mais nous avons observé que 


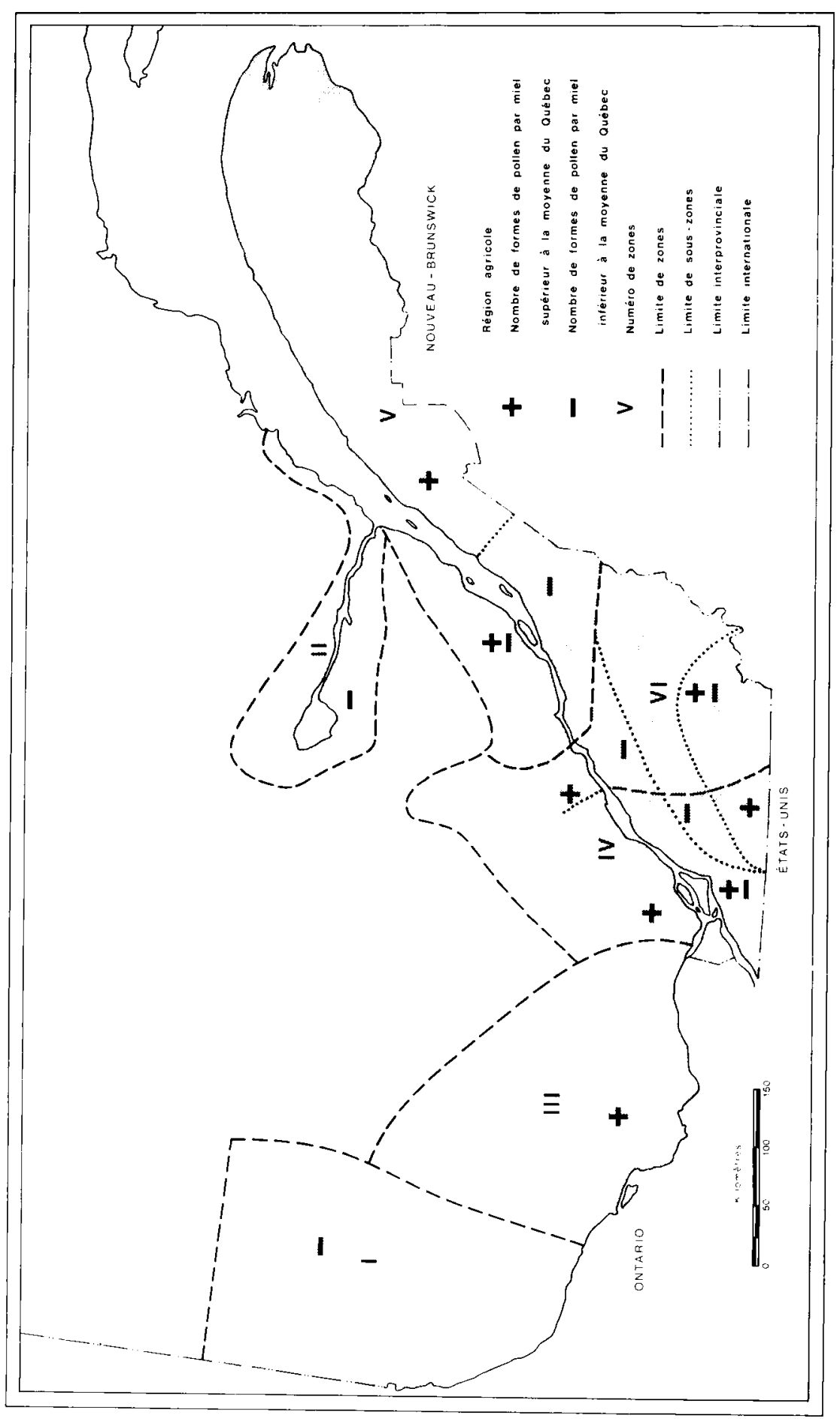


FIG. 4. - Cartographie des zones et des sous-zones et évaluation des nombres

de formes de pollen correspondants, par rapport à la moyenne québécoise.

$\div$ Nombre de formes de pollen par miel supérieur à la moyenne du Québec.

- Nombre de formes de pollen par miel inférieur à la moyenne du Québec.

\pm Nombre de formes de pollen oscillant autour de la moyenne du Québec.

Chiffre romain : numéro de zone (voir tableau 7 ).

Terre agricole

- _ Limite de zones

...... Limite de sous-zones

- - - Limite interprovinciale

Limite internationale

FIG. 4. - Maps of the zones and the sub-zones and evaluation of the number of pollen forms corresponding to the ratio of the average quebec sample.

$\div$ Number of pollen forms in honey greater than the average of Quebec

- Number of pollen forms in honey less than the average of Quebec

\pm Number of pollen forms similar to the average of Quebec

Roman numerals : number of the zone (see Table 7)

Agricultural land

- — - Border of the zones

$\ldots . .$. Border of the sub-zones

Interprovincial border

International border

l'importance et même la présence de certaines de celles-ci varient d'après les régions de la province et avons en outre décelé plusieurs pollens particuliers qui permettent de différencier les miels dans ce territoire.

\section{REMERCIEMENTS}

Nous adressons nos plus vifs remerciements à M. Yvan LAMONTAGNE du Laboratoire d'Expertise et d'Analyse alimentaire du Ministère de l'Agriculture à Québec qui nous a fourni la majorité des échantillons de miels et qui n'a pas cessé de nous prodiguer ses encouragements. Nous remercions également le Fonds Institutionnel de Recherche de l'Université du Québec à Rimouski qui a permis de mener ce travail à bien.

Reçu pour publication en décembre 1982

Eingegangen im Dezember 1982

\section{SUMMARY}

\section{POLLEN SPECTRUM OF QUEBEC HONEYS}

Microscopic pollen analysis was made from 164 honey samples collected from all the beekeeping areas of Quebec during 1977 and 1978 . In this study, $80 \%$ of the honey samples came from beekeeping areas different than those selected for a similar study conducted on 1974 and 1975 samples 
(Feller-Demalsy and Lamontagne, 1979). (Table 1 and Figure 1). The parallelism between the two studies showed that :

- the ratios of honeys with low and medium grain number have not significantly changed. Honeys with higher grain number were still uncommon (Table 2);

- varieties of pollen forms were observed less frequently in the Northern part of Quebec and in the areas where agriculture is extensively developed (region of Richelieu, Nicolet and Quebec South Shore) (Table 4 and Figure 3);

- Trifolium repens (white clover), Vicia cracca (cow vetch) and Solidago type (goldenrod) appeared to be stable and generally like the honey in Quebec;

- varieties of pollen forms like Rubus (raspberry), Fragaria (strawberry), Melilotus (honey clover) and Cruciferae were observed at a higher frequency. In the first two species, this can be attribued to the trend towards such cultivars, but for Melilotus and Cruciferae, the causes might be respectively a function of the climatic conditions and the resistance to herbicides;

- Vicia cracca (cow vetch) had to be added to the list of the plants of major importance resulting from the study made in 1974 and 1975;

- the regional features are precisely depicted (table 5,6 and 7);

- two new geographic indicators can be outlined : Rhus typhina (sumac) for the western part of the province and Acer spicatum (mountain maple) for the eastern area, especially in the Bas Saint-Laurent-Gaspésie region;

- the boundaries of the zones within Quebec resulting from the first honey study were retained and subdivised as seen in Figure 3 and Table 7. On a basis of pollen content in honey, Mauricie, Quebec and Nicolet can be reported as regions of transition between the eastern and western part of the province. A subdivision of the zones parallel to the Saint-Lawrence river was also investigated.

\section{ZUSAMMENFASSUNG}

\section{DAS POLLENSPEKTRUM DER HONIGE VON QUEBEC}

Die Pollenanalyse wurde an 164 Honigproben der Jahre 1977 und 1978 durchgeführt. Sie stammten aus den Hauptregionen von Quebec, in denen Bienenzucht betrieben wird. Die Herkunft der Proben ist zu $80 \%$ verschieden von denen, deren Analyse schon früher mit den Honigen der Jahre 1974 und 1975 durchgeführt worden war (Feller-Demalsy et LAMONTAGNE, 1979) (Tabelle 1 und Abb. 1). Der Vergleich der beiden Untersuchungen zeigt :

- die Anteile von Honigen mit geringer und mit mittlerer Pollenzahl sind ähnlich (Tab. 2); die Honige mit erhöhter Pollenzahl sind selten;

- die Zahl verschiedener Formen ist geringer einerseits in den nördlichsten Regionen, andererseits aber auch in den Regionen, mit dem höchsten Anteil kultivierten Landes (Tab. 4 und Abb. 3), d.h. in den Regionen von Richelieu, von Nicolet und von der Südküste von Quebec;

- Trifolium hybridum/repens (Hybrid- und Weißklee), Vicia cracca (Vogelwicke) und der Solidago-Typ (Goldrute) erscheinen als beständige und allgemeine Leitformen der Honige von Quebec;

- eine Vermehrung der bedeutenden Häufigkeiten betrifft mehrere Formen : Rubus (Himbeere), Fragaria (Erdbeere), Melilotus (Riesenhonigklee) und die Cruciferen; sie könnte für die beiden ersteren Arten ihre Ursache in der Intensivierung der betreffenden Kulturen haben, für Melilotus in Klimaschwankungen und für die Cruciferen in der Entstehung von Formen, die gegen selektive Herbizide resistent geworden sind;

- Vicia cracca nimmt in diesen Honigen eine so bedeutende Stellung ein, daß sie zu den wichtigsten Formen gezählt werden muB, die in der ersten Untersuchung zitiert worden sind;

- die regionalen Charakteristika wurden bestätigt und präzisiert (Tabellen 5, 6, 7); 
- zwei neue geographische Leitformen wurden aufgefunden, Rhus typhina (Essigbaum) für den Westen der Provinz und Acer spicatum (Ahorn) für den Osten, besonders für das Gebiet Bas Saint-Laurant-Gaspesie;

- die Zonen, die in der ersten Analyse der Honige von Quebec angedeutet wurden, konnten beibehalten und durch Aufteilung in Unterzonen weiter präzisiert werden (Abb. 3, Tab. 7); die Regionen von Mauricie, von Quebec und von Nicolet entsprechen hinsichtlich des Pollenbildes der Honige einer Übergangszone zwischen dem Osten und dem Westen der Provinz. Schließlich wurde noch eine Untergliederung der Zonen parallel zum Fluß Saint-Laurent in Erwägung gezogen.

\section{BIBLIOGRAPHIE}

BaILeY L. H., 1969. - Manual of Cultivated Plants. Mc Millan.

Bureau de la Statistique du Quebec, 1978. - Miels, Ministère de l'Industrie et du Commerce.

Butler G. and D. J. FinNey, 1945. - The influence of various physical and biological factors of the environment on honeybee activity. An examination of the relationship between activity and solar radiation. J. of exp. Biologi, 21, 206-212.

Снавот, 1948. - Plantes mellifères du Québec. Ministère de l'Agriculture. Service de l'Horticulture. Document non publié.

Chauvin R., ed. 1968. - Traité de Biologie de l'Abeille. Tome III Les produits de la ruche. Masson et Cie. Paris.

Commision De Toponymie, 1978. - Répertoire toponymique du Québec. Editeur officiel du Québec.

EMOND Gilles, 1981. - Situation historique et actuelle de la malherbologie au Québec et perpective d'avenir. Bulletin de l'Acfas, 3, (2), 15-20.

Erdtman G., 1972. - Pollen Morphology and Plant Taxonomy. Hafner Publishing Comp.

Feller-Demalsy M. J. et Y. Lamontagne, 1979. - Analyse pollinique des miels du Québec. Apidologie, 10 (4), 313-340.

FERnald M. L., 1950. - Gray's Manual of Botany. American Book Company.

Ferron M. et R. CayouetTe, 1975. - Nom des mauvaises herbes du Québec. QA38 R4-4. Agriculture Québec.

HAMBleton J. I., 1940. - The effect of weather upon the changes in weight of a colony of bees during the honeyflow. Journal of Economic Entomoloby, 33, 865-870.

Labrecque J. et M. Thibault, 1978. -- Atlas l'Agro-alimentaire au Québec. Gouvernement du Québec. Ministère de l'Agriculture.

Lieux M. H., 1972. - A melissopalynological study of 54 Louisiana (USA) honeys. Rev Palaeobot. Palynol, 13, 94-124.

Louveaux J., 1966. - Pollenanalyse einiger kanadischer Honige. Zeitschrift für Bienenforschung, 8, 195-202.

Louveaux J. et P. Vergeron, 1964. - Étude du spectre pollinique de quelques miels espagnols. Ann. Abeille, 7 (4), 329-347.

Louveaux J., Maurizio A, Vorwohl G., 1970. - Methods of Melissopalynology. Bee World, 51, 125-128.

MARIE-VICTORIN Frère, 1964. - Flore laurentienne. Presses de l'Université de Montréal.

Maurizio A., 1951. - Pollen analysis of Honey. Bee world, 32, 1-5.

Maurizio A., 1971. - Le spectre pollinique des miels luxembourgeois. Apidologie, 2, 221-238.

Maurizio A. et Louveaux J., 1965. - Pollens de plantes mellifères d'Europe. Union des groupements apicoles français. Paris.

Ministère de L'agriculture, 1974. - Atlas L'Agro-alimentaire au Québec. Gouvernement du Québec. Moore P. D. and Wesb J. A., 1978. - An Illusrated guide to Pollen Analysis. Halsted Press. New York. 
Nilson S., PraglowkI J. and NiLsON L., 1977. - Atlas of airborne pollen grains and spores in northern Europe. Ljungföretagen. Orebro. Sweden.

Oertel E., 1939. - Honey and pollen-plants of the United States. United States Department of Agriculture. Circular, no 554.

Pellet F. C., 1977. - American Honey Plants. Dadant and Sons Publication.

RichaRd P., 1970. - Atlas pollinique des arbres et de quelques arbustes indigènes du Québec. Le naturaliste Canadien, 97, 97-162.

Roland A. E. and E. C. Smith, 1969. - The flora of Nova Scotia, Nova Scotia Museum. Halifax.

Rousseau C., 1968. - Histoire, habitat et distribution de 220 plantes introduites au Québec. Naturaliste Can, 95, 49-171.

RousseaU C., 1974. - Géographie floristique du Québec/Labrador. Distribution des principales espèces vasculaires. Les presses de l'Université Laval.

SERVice DE LA MÉtÉorologié, 1975. - Bulletin météorologique. Gouvernement du Québec. Ministère des Richesses naturelles. Direction générale des eaux, 14 (7), 1-27.

SERVICE DE LA MÉTÉOROLOGIE, 1978. - Bulletin météorologique. Gouvernement du Québec. Ministère des Richesses naturelles. Direction générale des eaux, 14 (7), 1-22.

Service des EtUdes economiques, 1978. - Coup d'ail sur l'agro-alimentaire au Québec. Ministère de l'Agriculture du Québec.

SCoGgan H. J., 1978. - The flora of Canada. National Museum of Canada.

StatistiQue CANADA, 1979. - Recensement du Canada. 1976. Agriculture. Illustration graphique. Catalogue, 96-871.

Vorwohl G., 1971. - Importance et but de l'analyse pollinique du miel. Apiacta, 2, 51-54.

WILSON C. V., 1971. - Le climat du Québec, Ire partie. Atlas climatique. Service de la météorologie du Canada. 